\title{
Nonlinear thermomechanical response and constitutive modeling of viscoelastic polyethylene membranes
}

\author{
F. Bosi ${ }^{a, *}$, S. Pellegrino ${ }^{a}$ \\ ${ }^{a}$ Graduate Aerospace Laboratories, California Institute of Technology, Pasadena, CA, 91125, USA
}

\begin{abstract}
Thin films of linear low-density polyethylene show a significant time-dependent behavior, strongly reliant on temperature and strain rate effects. A constitutive nonlinear thermo-viscoelastic relation is developed to characterize the response of thin membranes up to yielding, in a wide range of temperatures, strain rates, and mechanical loading conditions. The presented plane stress orthotropic formulation involves the free volume theory of viscoelasticity and the time-temperature superposition principle, necessary to describe non-linearities and non-isothermal conditions. Uniaxial tension tests at constant strain rate and long-duration biaxial inflation experiments have been employed in the calibration of the material parameters. The model has been implemented in the Abaqus finite element code by means of a user-defined subroutine based on a recursive integration algorithm. The accuracy of the constitutive relation has been validated against experimental data of full field diaphragm inflation tests and uniaxial tension, relaxation and cyclic experiments, covering sub-ambient temperatures and strain rate ranges observed during the operation of stratospheric balloons.
\end{abstract}

Keywords: Nonlinear Viscoelasticity, Thin Films, Free Volume Model, Polymers, Balloons

\section{Introduction}

Polymeric thin films are widely used as structural elements in biomedical devices, civil structures, tissue engineering and aerospace systems due to their ability to undergo large elastic deformations when implemented in lightweight components. In practical applications, these elements are subjected to different thermal conditions, loading rates, and cyclic deformations. Therefore an accurate description of their response at different temperatures and strain rates is fundamental to a successful design of membrane structures.

For aerospace missions, stratospheric balloons have been employed to observe scientific phenomena in space and on Earth. They are realized with a polyethylene sealed membrane stiffened by meridional tendons (Smith and Rainwater, 2004; Cathey, 2009). In the past years, research efforts were focused on the balloon inflation in order to avoid instabilities which were leading to an incomplete deployment of the structure (Wakefield, 2005. Pagitz and Pellegrino, 2010). Nowadays, a design of cleft-free balloons is possible through a numerical clefting test (Deng and Pellegrino 2011). In order to prevent the failure of the polymeric envelope and achieve longer flights, an adequate model is required to describe the behavior of this material, which shows pronounced time-dependent effects during day-night pressurization cycles at sub-ambient temperatures (Rand et al. 1996). Considering the need of an accurate prediction of the structural response, the aim of the present study is to develop a nonlinear thermoviscoelastic constitutive relation for anisotropic polyethylene

\footnotetext{
${ }^{*}$ Corresponding author

Email addresses: fbosi@caltech.edu (F. Bosi), sergiop@caltech.edu (S. Pellegrino)
}

membranes, based on a detailed experimental characterization.

Many models have been developed to describe the viscoelasticity of polymeric materials. A nonlinear viscoelastic equation based on a single integral formulation was derived from thermodynamic principles and firstly introduced by Schapery (1966. 1969). The extension of this theory was proposed by Knauss and Emri (1981) who postulated that the variations in the intermolecular space is related with the macroscopic material response (free volume model). An alternative approach modeled the temperature and rate-dependent behavior of polymers through a two mechanisms process, namely intermolecular interactions and network interactions (Bergstrom and Boyce, 2001, Dupaix and Boyce, 2007). The former mechanism is described as a thermally activated process where segments of chain molecules exceed a potential energy barrier (Ree and Eyring, 1955).

The thin film that constitutes the NASA Superpressure balloon envelope is produced as a three layer extrusion of Linear Low Density Polyethylene (LLDPE), with a total thickness of $38 \mu \mathrm{m}$. The extrusion process consists of pulling the resin from a circular die along the machine direction (MD) and simultaneously stretching it in the transverse direction (TD). The amount of stretching is quantified through the bubble-to-die diameter, called blow-up-ratio (BUR), which is equal to two in order to achieve nearly balanced properties between the two directions (Yoon and Park, 2000). An ultraviolet inhibitor is added to the outer layer of the film in order to protect the inner layer from radiation damage. Since the glass transition temperature of this material is ca. $-95^{\circ} \mathrm{C}$ and the operational range of the balloon is between ambient temperature and $-80^{\circ} \mathrm{C}$, LLDPE is in the rubbery state during flight. LLDPE was first modeled on the basis of Schapery's theory considering orthotropy and 
biaxial loading effects (Rand and Sterling, 2006). This model was implemented in a finite element software and compared with experiments on pressurized cylinders, showing good agreement at small strains. In order to capture the large deformation behavior, Li et al. (2016) combined a linear viscoelastic model based on experimentally derived master curves with the free volume theory. They developed a constitutive relation for LLDPE film for short duration uniaxial and biaxial tests between $+10^{\circ} \mathrm{C}$ and $-50^{\circ} \mathrm{C}$.

The present study wants to extend the validity of the previous model ( $\mathrm{Li}$ et al., 2016) to the wider range of operational conditions the material will experience during flight. Therefore a longer time domain with slower deformation rates, a wider temperature range (including ambient temperature), and different loading conditions (relaxation and cyclic tests) will be considered. A constitutive relation extension is required because the previous model was calibrated by means of experiments performed at a strain rate $\dot{\epsilon}=0.1 \% \mathrm{~s}^{-1}$, while the inflation rate of the balloon is ca. two orders of magnitude lower. The predictions of the existing constitutive model are underestimating the experimental stress values by $30-50 \%$ for low temperatures $\left(T \leq-10^{\circ} \mathrm{C}\right)$ and low strain rates $\left(\dot{\epsilon} \leq 0.01 \% \mathrm{~s}^{-1}\right)$. Furthermore, the material parameters must be calculated considering deformation mechanisms during long-term experiments, since the previous model is too stiff when constant pressure is applied for several hours. For this purpose, long-duration membrane inflation tests are carried out in this work. The model extension to ambient temperature, when the material behaves more similarly to a viscous fluid, is fundamental for the accurate description of the first inflation phase of the balloon during launch, while cyclic tests are necessary to evaluate the constitutive relation performance with loading and unloading phases, as in day-night cycles during stratospheric flight. The new experiments are performed within the viscoelastic domain $(\epsilon<6 \%)$, avoiding the onset of plasticity by monitoring the residual deformation after strain recovery, a feature which was not considered in the development of the previous model. In particular, uniaxial tensile tests were performed until the yield point of the material. Lastly, in order to be readily employed in any structural analysis, this study presents a numerical implementation of the constitutive relation into a commercial finite element software. The paper is organized as follows: Sect.2presents the mathematical model of nonlinear thermo-viscoelasticity for plane stress regime based on the free volume theory, while Sect. 3 shows the experimental procedures and results that were adopted in the calibration of the material parameters in Sect. 4. Sect. 5 presents the finite element implementation of the proposed model. Lastly, numerical predictions are compared with experimental measurements at different temperatures, strain rates and loading conditions in Sect.6

\section{Constitutive model}

Polyethylene thin film shows a pronounced time-dependent behavior that can be described by means of the nonlinear theory of thermo-viscoelasticity, based on the free volume model. Following $\mathrm{Li}$ et al. (2016), the membrane is modeled as an orthotropic material under plane stress conditions, where the inplane axes are denoted with 1 and 2, representing the directions MD and TD respectively, the out-of-plane axis is denoted with subscript 3, while 6 represents the shear component.

The thermo-viscoelastic constitutive relation is Brinson and Brinson, 2008,

$$
\boldsymbol{\epsilon}(t)=\int_{0}^{t} \boldsymbol{D}(t-s) \dot{\boldsymbol{\sigma}} \mathrm{d} s+\int_{0}^{t} \alpha \dot{T} \mathrm{~d} s,
$$

where $\dot{(}) \equiv \mathrm{d}() / \mathrm{d} s$ is the time derivative. The viscoelastic model is valid until the onset of plasticity and in this range the strains are considered small, hence the model is developed in terms of engineering strain $\epsilon$ and engineering stress $\sigma$, whose components are reported together with the coefficient of thermal expansion (CTE) $\boldsymbol{\alpha}$

$$
\boldsymbol{\epsilon}(t)=\left[\begin{array}{c}
\epsilon_{1}(t) \\
\epsilon_{2}(t) \\
\epsilon_{3}(t) \\
\epsilon_{6}(t)
\end{array}\right], \quad \boldsymbol{\sigma}(t)=\left[\begin{array}{c}
\sigma_{1}(t) \\
\sigma_{2}(t) \\
0 \\
\sigma_{6}(t)
\end{array}\right], \quad \boldsymbol{\alpha}=\left[\begin{array}{c}
\alpha_{1} \\
\alpha_{2} \\
\alpha_{3} \\
0
\end{array}\right] .
$$

Since it is not possible to experimentally assess the out of plane thickness $\epsilon_{3}$, the small strain hypothesis does not require any further assumptions, such as incompressible behavior, for the empirical determination of the stresses, Sect. 3. It can be observed that the constitutive model is a plane stress relation with the presence of a non-zero out-of-plane deformation $\epsilon_{3}$, while $\sigma_{3}$ is always zero. Therefore, the creep compliance matrix in Eq. (1) is written as

$$
\boldsymbol{D}(t)=\left[\begin{array}{cccc}
D_{11}(t) & D_{12}(t) & D_{13}(t) & 0 \\
D_{12}(t) & D_{22}(t) & D_{23}(t) & 0 \\
D_{13}(t) & D_{23}(t) & 0 & 0 \\
0 & 0 & 0 & D_{66}(t)
\end{array}\right]
$$

Each compliance term $D_{i j}(t)$ in Eq. (3) is represented by a Prony series of $n$ exponential functions

$$
D_{i j}(t)=D_{i j, 0}+\sum_{k=1}^{n} D_{i j, k}\left(1-e^{-t / \tau_{k}}\right),
$$

where $t$ is time, $D_{i j, 0}$ is the instantaneous compliance at $t=0$, $D_{i j, k}$ are the Prony coefficients representing the variation of creep compliance over a certain time domain, and $\tau_{k}$ is the retardation time corresponding to the $k$-th element. The time-temperature superposition principle (TTSP) defines the reduced time $t^{\prime}$ for non-isothermal conditions (Schapery, 1969) as

$$
t^{\prime}=\int_{0}^{t} \frac{\mathrm{d} s}{a(T(s), \boldsymbol{\epsilon}(s))},
$$

where $a(T(s), \boldsymbol{\epsilon}(s))$ is the shift factor, which is less (more) than unity to simulate a delayed (accelerated) viscoelastic process for $T<T_{0}\left(T>T_{0}\right)$. Nonlinearities of the model are taken into account considering an expression of the shift factor that depends on the variation in the intermolecular space within the polymer, known as free volume model (Knauss and Emri, 1987). The fractional free volume is defined as

$$
f=f_{0}+\alpha_{v}\left(T-T_{0}\right)+\delta_{v} \theta+\delta_{s} \epsilon_{\mathrm{eff}},
$$


where $f_{0}$ is the fractional volume in the reference, unstressed state at temperature $T_{0}, \alpha_{v}=\alpha_{1}+\alpha_{2}+\alpha_{3}$ is the volumetric coefficient of thermal expansion, $\delta_{v}$ and $\delta_{s}$ are constants relating strain quantities variations to changes in free volume, $\theta=\sum_{k=1}^{3} \epsilon_{k}$ is the mechanical dilatation, while $\epsilon_{\text {eff }}$ represents the effective deviatoric mechanical strain

$$
\epsilon_{\mathrm{eff}}=\sqrt{\frac{2}{3}\left[\sum_{k=1}^{3}\left(\epsilon_{k}-\frac{\theta}{3}\right)^{2}+\kappa \epsilon_{6}^{2}\right]},
$$

where $\kappa$ is a distorsional parameter to be experimentally determined. The time shift factor is expressed in terms of the free volume $f$

$$
\log a=\frac{B}{2.303 f_{0}}\left(\frac{f_{0}-f}{f}\right),
$$

where $B$ is a material constant. The constitutive relation in Eq. (1) can be finally rewritten, considering temperature variations through the new time scale in Eq. (5), as

$$
\boldsymbol{\epsilon}\left(t^{\prime}\right)=\int_{0}^{t^{\prime}} \boldsymbol{D}\left(t^{\prime}-s\right) \dot{\boldsymbol{\sigma}} \mathrm{d} s+\int_{0}^{t^{\prime}} \alpha \dot{T} \mathrm{~d} s .
$$

\section{Experimental procedures and results}

This section presents the experiments performed on LLDPE film at different temperatures and strain rates. They include uniaxial tests (tensile, relaxation and cyclic) and diaphragm inflation experiments. The latter, together with uniaxial tensile tests at $0.001 \% \mathrm{~s}^{-1}$ of strain rate, will be used in the calibration of the material parameters presented in Sect. 4. The remain experiments will serve as a validation of the accuracy of the constitutive model in Sect. 6 .

Experiments were conducted with an Instron 3119-506 environmental chamber, mounted on an Instron 5569 electromechanical testing machine equipped with an Instron 2525-816 load cell (R.C. 500N). Two type-K thermocouples were placed as close as possible to the specimen, on the top and bottom of the two grips and on the aluminum upper ring, for uniaxial and diaphragm tests respectively. The temperature was acquired through an Omega HH802U thermocouple reader and Omega HH800SW software, showing oscillation within $\pm 0.3^{\circ} \mathrm{C}$ once a constant temperature had been reached. The experimental setup for both uniaxial and biaxial tests is shown in Fig. 1.

The VIC3D ${ }^{\mathrm{TM}}$ (Correlated Solution, v.7) non-contact measurement system was used to measure full-field displacements and strains by means of a three-dimensional Digital Image Correlation system (Sutton et al. 2009). A pair of stereo-mounted digital cameras Grasshopper 5.0 MP Mono FireWire 1394b (PointGrey) equipped with Schneider Kreuznach Xenoplan lenses (Focal length 35 mm, F-number 8), and VIC-Snap8 software were employed to acquire images. Cameras set-up and samples preparation were determined in order to minimize uncertainties and noise level during image postprocessing (Reu, 2012). For this reason, a stereo-angle of ca. $25^{\circ}$ was chosen (camera distance of $26 \mathrm{~cm}$, specimen-camera distance of $60 \mathrm{~cm}$ ). Black paint was lightly sprayed on the transparent samples in order
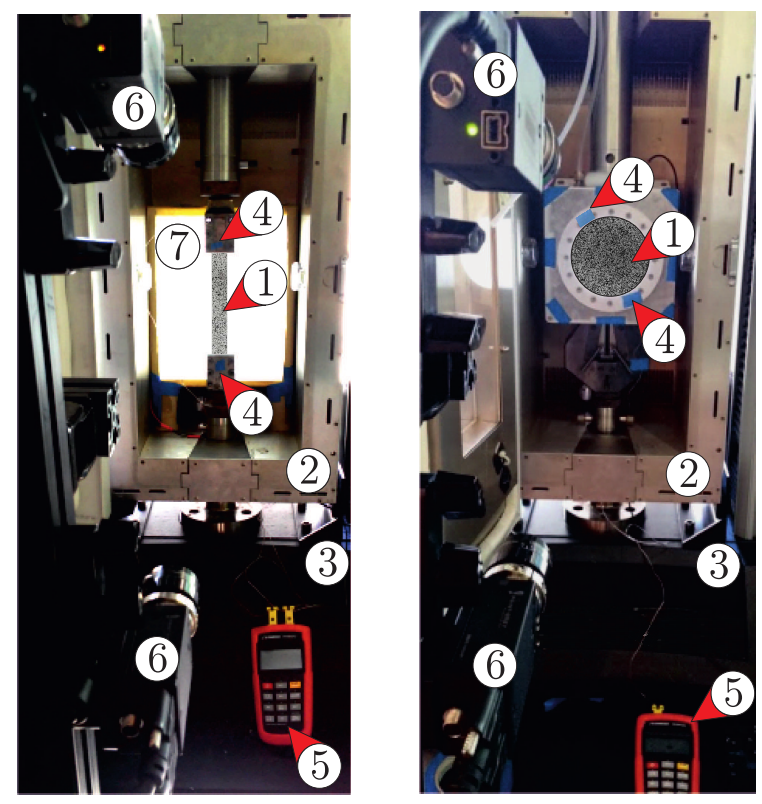

Figure 1: Experimental setup for uniaxial (left) and biaxial (right) tests, realized with a LLDPE specimen (1) placed inside an environmental thermal chamber (2) mounted on an Instron testing machine (3). Thermocouples (4), thermocouple reader (5) and stereo-cameras (6) were employed to acquire temperatures and images during experiments.

to provide a random speckle pattern, with an average speckle dimension of 6 pixels, necessary to avoid aliasing. Adequate contrast was achieved using white LED lights underneath the specimen. The calibration of the stereo system relates the physical 3D world to a 2D camera image via a mathematical model by means of intrinsic and extrinsic parameters. In order to obtain a calibration score lower than 0.03 pixels, more than 25 static images per camera were acquired. The calibration images were obtained by tilting, rotating and translating the calibration target $(12 \times 9$ dots, spacing $5 \mathrm{~mm})$ throughout the entire field of view.

Experiments were performed below the yield point of the material, carefully checking that after each test the residual strain was below $0.2 \%$.

\subsection{Uniaxial tests}

Uniaxial tensile, relaxation and cyclic tests were performed on rectangular specimens of dimension $200 \mathrm{~mm} \times 25.4 \mathrm{~mm}$, cut with a JDC Precision Cutter (Thwing-Albert Inc.). In order to quantify the material orthotropy, experiments were carried out with the sample aligned along both machine (MD) and transverse (TD) directions. For each temperature and strain rate considered, the results presented in the following are an average of three tests. During the postprocessing, a correlation subset of $31 \times 31$ pixels was chosen to achieve enough spatial resolution. A step size of 7 pixels and a filter size of 13 pixels were adopted to maintain a good point density. In order to guarantee a high sub-pixel accuracy, Gaussian weights, 8-tap splines interpolation, and zero-normalized square difference minimization functions were selected during image processing. As a result, the projection error, which is a measure of correlation accuracy, was always 


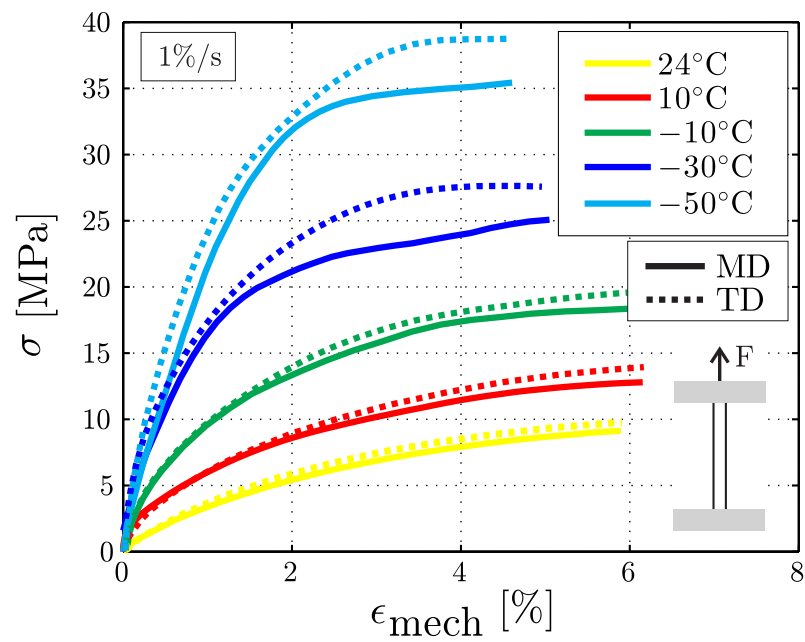

Figure 2: Nominal stress vs. mechanical engineering strain during uniaxial tension tests at $\dot{\epsilon}=1 \% \mathrm{~s}^{-1}$ and $T=[+24 ;+10 ;-10 ;-30 ;-50]^{\circ} \mathrm{C}$. Samples were aligned with respect to MD (solid line) and TD (dashed line).

below 0.07 pixels. Since the DIC reference image was taken after the sample had been cooled, the measured strain field is the mechanical part of the total strain, while thermal deformations were not measured.

\subsubsection{Tensile tests}

Uniaxial tension tests at constant strain rate were performed to characterize the nonlinear behavior of the film in the rubbery state, between room temperature $\left(24^{\circ} \mathrm{C}\right)$ and the glass transition temperature (ca. $-95^{\circ} \mathrm{C}$ ). For each of the five temperatures selected, namely $T=[+24 ;+10 ;-10 ;-30 ;-50]^{\circ} \mathrm{C}$, tests were carried out at four strain rates, $\dot{\epsilon}=[1 ; 0.1 ; 0.01 ; 0.001] \% \mathrm{~s}^{-1}$. The temperature was reached by cooling the environmental chamber with the sample in a slack and unstressed state for $30 \mathrm{~min}$ (20 min to achieve the desired temperature and $10 \mathrm{~min}$ to stabilize it within $\pm 0.3^{\circ} \mathrm{C}$ ). The bottom end of the sample was held fixed, whereas the top end was attached to the moving beam of the Instron machine, which provided the nominal strain rate of the test. The engineering stress was computed from the measured load divided by the initial cross sectional area of the sample $(25.4 \mathrm{~mm} \times 0.038 \mathrm{~mm})$. During the test, the load was acquired through a NI USB-6221 data acquisition module (DAQ), and it is reported in Fig. 2 as a function of the nominal strain for different temperatures. For conciseness, only the results at $\dot{\epsilon}=1 \% \mathrm{~s}^{-1}$ are presented; they show a highly nonlinear behavior of the material. This response justifies the choice of the shift factor $a$ to be dependent on the free volume, in order to correctly capture nonlinearities. It can be noticed that the transverse direction (TD) is stiffer than the machine direction (MD) because of the co-extrusion manufacturing process which leads to different material properties along the two stretching directions. This response requires an orthotropic model, so that $D_{11} \neq D_{22}$ and $D_{12} \neq D_{13} \neq D_{23}$. As expected for polymers, the material becomes stiffer for lower temperatures.

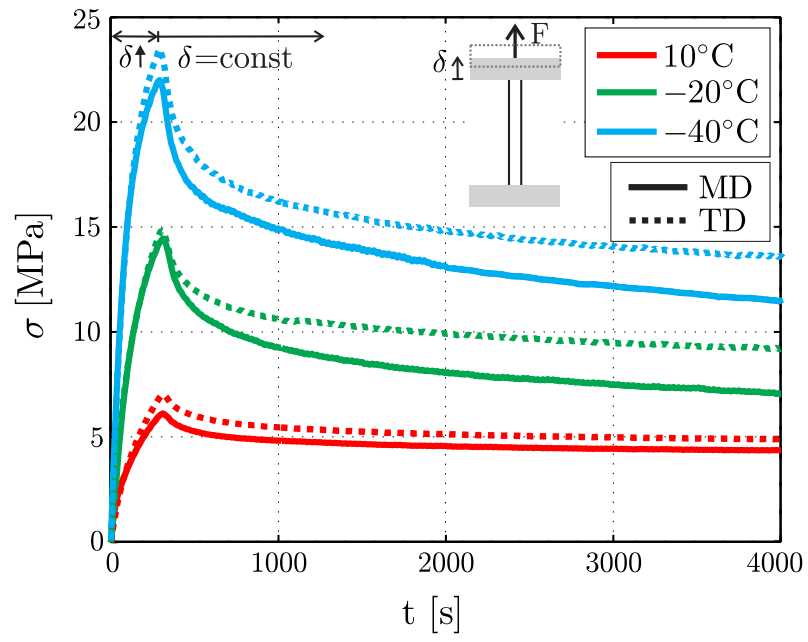

Figure 3: Nominal stress vs. time for relaxation tests performed at $T=$ $[+10 ;-20 ;-40]^{\circ} \mathrm{C}$ for both MD (solid line) and TD (dashed line). The sample was elongated at $\dot{\epsilon}=0.01 \% \mathrm{~s}^{-1}$ for $300 \mathrm{~s}$, then the longitudinal mechanical strain was kept constant at $3 \%$ for $3700 \mathrm{~s}$.

\subsubsection{Relaxation tests}

Uniaxial relaxation tests were carried out for both MD and TD directions at $T=[+10 ;-20 ;-40]^{\circ} \mathrm{C}$ to provide the validation data for the model, Sect. 6. These experiments were performed in two stages; first the sample was loaded and then it was kept under tension at a fixed displacement. In the first phase, the crossbeam of the Instron machine was moved at a speed of $0.002 \mathrm{~mm} / \mathrm{s}$ for $300 \mathrm{~s}$ in order to achieve a nominal strain rate of $0.01 \% \mathrm{~s}^{-1}$. During the second phase, the sample was kept under tension at $3 \%$ of longitudinal mechanical strain for $3700 \mathrm{~s}$, to characterize the stress relaxation behavior of the film. Experimental results are presented in Fig. 3. where it can be observed that the direction MD is softer than TD. Moreover, with a decrease in temperature, the material is stiffer and the stress relaxation is more pronounced.

\subsubsection{Cyclic tests}

Polymeric membranes are often subjected to multiple tensile loading and unloading phases, especially during day-night cycles in balloon flights. Therefore, it is important to investigate the film response in a tensile cyclic experiment in order to characterize hysteresis and stress softening behavior. Tests were run for both MD and TD directions at room temperature $\left(24^{\circ} \mathrm{C}\right)$ and $-10^{\circ} \mathrm{C}$. The sample was loaded and unloaded at $\dot{\epsilon}=0.1 \% \mathrm{~s}^{-1}$ until a maximum mechanical strain of $3 \%$. The measured nominal stress as a function of the crossbeam displacement is reported in Fig. 4. As noticed in the previous uniaxial tests, the material is stiffer along the transverse direction TD and with a decrease of temperature. Furthermore, hysteresis and stress softening are enhanced at lower temperatures, while no substantial difference can be found between MD and TD.

\subsection{Diaphragm inflation tests}

Long-duration biaxial tests were carried out inflating a $100 \mathrm{~mm}$ diameter membrane in an air pressure box at $T=$ 


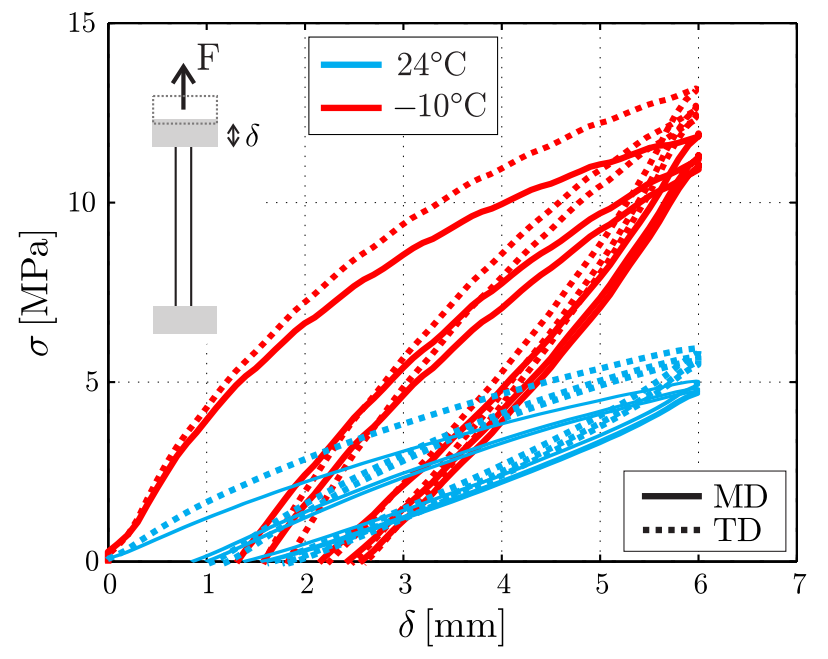

Figure 4: Nominal stress vs. longitudinal displacement for cyclic tests at $\dot{\epsilon}=0.1 \% \mathrm{~s}^{-1}$. Experiments performed at $24^{\circ} \mathrm{C}$ (blue) and $-10^{\circ} \mathrm{C}$ (red) are shown for both MD (solid curve) and TD (dashed curve).

$[+24 ;+10 ; 0 ;-10 ;-30 ;-50]^{\circ} \mathrm{C}$. The pressure signal was generated with LabView SignalExpress (v.2.5.1) and a National Instruments (NI) USB-6221 DAQ, connected to an analog AD694JN voltage-to-current converter and an Omega IP610-030 pressure controller. An Omega DPG409-015G electronic pressure gauge was interfaced with the NI DAQ device and Vic-Snap software (v.8) in order to measure the inlet pressure.

The test sample was fixed to the box applying a tiny pretension (maximum pre-strain was $<0.1 \%$ ) in order to guarantee a flat initial surface without wrinkles. For experiments performed at sub-ambient temperatures, during the $30 \mathrm{~min}$ of cooling an initial constant overpressure (ca. $1300 \mathrm{~Pa}$ ) was applied to the sample in order to maintain positive Gaussian curvature throughout the specimen. After the desired temperature had been reached, the pressure was increased to achieve a constant strain rate for $500 \mathrm{~s}$, until the mechanical strain at the apex of the inflated diaphragm was between $2 \%$ and $3 \%$. Once the maximum pressure had been reached, it was kept constant for 2 hours by sending a constant voltage signal to the pressure gauge through Labview SignalExpress.

DIC postprocessing was performed considering a circular area of interest (AOI) of $90 \mathrm{~mm}$ diameter centered at the apex of the film. The edges of the sample were excluded because edge shadowing led to poor correlation. The subset dimension was $33 \times 33$ pixels, with a step size of 7 pixels; a filter size of 11 and 25 pixels was chosen for strains and curvatures, respectively. The reference image was taken at the beginning of the test, at room temperature, therefore the strains measured by DIC were the total strains. The mechanical strain components were calculated during postprocessing by subtracting the thermal strain, Eq. 9). Displacements and curvatures were measured throughout the whole AOI, while the nominal stress was calculated at the apex of the diaphragm through the isotropic assumption

$$
\bar{\sigma}=\frac{p \bar{R}}{2 h},
$$

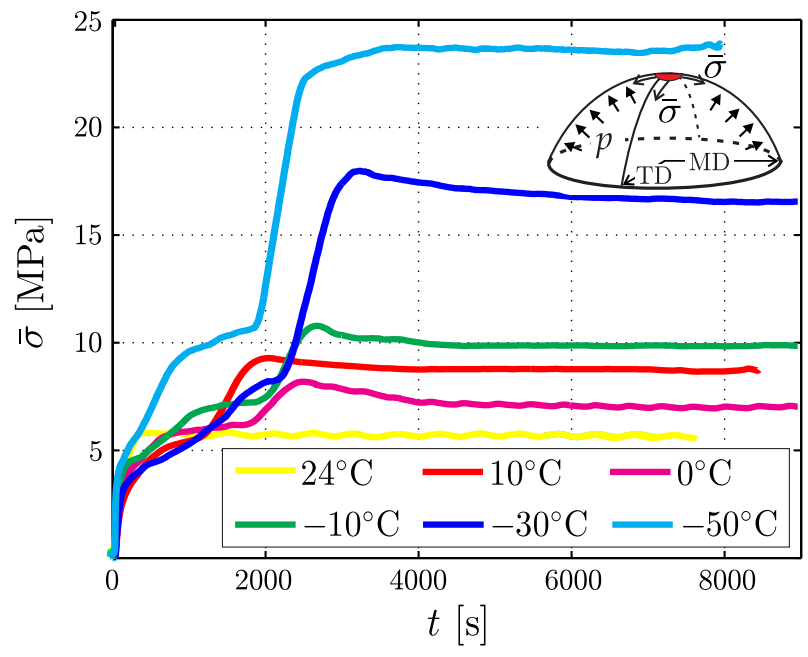

Figure 5: Diaphragm inflation tests of circular $100 \mathrm{~mm}$ diameter sample at different temperatures, namely $T=[+24 ;+10 ; 0 ;-10 ;-30 ;-50]^{\circ} \mathrm{C}$. Nominal stress at the apex of the bubble, calculated through Eq. [10, is reported as a function of time.

where $p$ is the inlet measured pressure, $h$ is the initial thickness of the film $(38 \mu \mathrm{m})$ and $\bar{R}$ is the mean radius of curvature between the two principal directions MD and TD. This assumption has been validated in Sect. 6.2 The projection error during image correlation was always below 0.09 pixels, showing a very good correlation despite the presence of the thermal chamber's window between the cameras and the test sample.

The maximum nominal stress in the film is shown in Fig. 5 as a function of time, for tests at $T=[+24 ;+10 ; 0 ;-10 ;-30 ;-50]^{\circ} \mathrm{C}$. Results from biaxial experiments were employed to calibrate the material parameters of the model in Sect. 4, while a detailed comparison between numerical predictions and experimental data is presented in Sect. 6.2

\section{Determination of material parameters}

The constitutive model outlined in Sect. 2 requires several parameters to be determined from experimental observations. They are the six creep compliance coefficients $\left[D_{11} ; D_{12} ; D_{22} ; D_{13} ; D_{23} ; D_{66}\right]$ and the five free volume model parameters $\left[B ; f_{0} ; \delta_{v} ; \delta_{s} ; \kappa\right]$ that take into account the nonlinearity of the material. Furthermore, the coefficients of thermal expansion $\left[\alpha_{1} ; \alpha_{2} ; \alpha_{3}\right]$ are necessary to compute the thermal strains and the volumetric coefficient of thermal expansion $\alpha_{v}$, Eq. (6). Extensive studies of LLDPE CTEs have been performed by Young (2010) and Li et al. (2016). The former proposed a polynomial representation of the in-plane coefficients $\alpha_{1}$ and $\alpha_{2}$

$$
\alpha_{i}=\sum_{j=0}^{12} \rho_{i, j} T^{j}, \quad i=1,2
$$

where $\rho_{i, j}$ are the polynomial coefficients listed in Table A.1 and $T$ is the temperature expressed in $\mathrm{K}$. The latter showed that a representative value of the out-of-plane CTE is $\alpha_{3}=10^{-4} \mathrm{~K}^{-1}$. 
The in-plane creep compliance coefficients $\left[D_{11} ; D_{12} ; D_{22}\right]$ were obtained from creep test data by $\mathrm{Li}$ et al. (2016) and are reported in Table A.2. A single set of relaxation times $\tau_{k}$ was selected for all compliance coefficients, with time interval of one decade in order to cover the entire temporal range of the master curve and achieve enough smoothness for the interpolating Prony series (Park and Kim, 2001).

Therefore, the remaining material parameters, still to be determined are the free-volume model parameters $\left[B ; f_{0} ; \delta_{v} ; \delta_{s} ; \kappa\right]$ and the creep compliances $\left[D_{13} ; D_{23} ; D_{66}\right]$. Each of these parameters requires $n=19$ Prony coefficients. To avoid non-uniqueness in the determination of both $\delta_{v}$ and $\epsilon_{v}$ from the out-of-plane compliances $D_{13}$ and $D_{23}, \delta_{v}=1$ has been assumed, thus the total number of unknowns is 65 . The high number of material parameters is mainly due to the number $n=19$ of exponential functions in the Prony series, Eq. (4). A reduction of $n$ leads to a poor approximation of the master curves and to an inadequate prediction of the behavior of complex materials such as LLDPE films.

Following the optimization procedure developed by Li et al. (2016), the model fitting consists of a two-stage calibration process, where the first step is an optimization of the unknown $\boldsymbol{x}=\left[B ; f_{0} ; \delta_{s} ; D_{13} ; D_{23}\right]$ through the minimization of the following functional

$$
F(\boldsymbol{x})=\sum_{j \in \mathfrak{U}} \omega_{\mathcal{U}}\left(\hat{y}_{j}(\boldsymbol{x})-y_{j}\right)^{2}+\sum_{j \in \mathfrak{D}} \omega_{\mathcal{D}}\left(\hat{y}_{j}(\boldsymbol{x})-y_{j}\right)^{2},
$$

where $y_{j}$ and $\hat{y}_{j}$ are the $j$-th stress data points for experiments and model prediction respectively, taken from a set of uniaxial tension tests $\mathcal{U}$ and diaphragm inflation experiments $\mathcal{D}$. The uniaxial tests considered in the determination of the material coefficients were all the experiments performed at $\dot{\epsilon}=0.001 \% \mathrm{~s}^{-1}$. These tests capture the material behavior at different temperatures and at a strain rate representative of the deformation rate the polymeric film experiences during a balloon flight. The biaxial tests included in the set $\mathcal{D}$ are those presented in Fig. 5 and they characterize the response of the film in a biaxial stress state, characteristic of balloon structures. In the minimization of Eq. 12, different weight factors have been employed. For uniaxial tests and the cooling, loading and constant pressure phases of biaxial experiments the weight factor was $\omega_{\mathcal{U}}=\omega_{\mathcal{D}}=1$. Whereas, for the pressurization phase at room temperature of the diaphragm tests, the weight coefficient was $\omega_{\mathcal{D}}=0.1$.

The minimization of the functional $F(\boldsymbol{x})$ was treated as a constrained optimization problem, since the free volume parameters $\left[B ; f_{0} ; \delta_{s}\right]$ must be strictly positive, while all the Prony series coefficients for $D_{13}$ and $D_{23}$ have to be negative in order to avoid an unstable material behavior, related to waviness of the master curves. An interior-point algorithm, implemented through the MATLAB (R2015a) function fmincon provided a fast convergence to the best set of model coefficients, using the parameters in Li et al. (2016) as initial guess.

The second step of the calibration consists in determining the Prony series coefficients of $D_{66}$ and the parameter $\kappa$, which is a distorsional constant related to the shear strain in Eq. (7). The optimization of these parameters is independent from the
Table 1: Prony coefficients for compliance matrix $\boldsymbol{D}\left[\mathrm{MPa}^{-1}\right]$

\begin{tabular}{ccccc}
\hline $\mathbf{j}$ & $\tau[\mathrm{s}]$ & $-D_{13, j}$ & $-D_{23, j}$ & $D_{66, j}$ \\
\hline 0 & 0 & $1.4242 \cdot 10^{-6}$ & $1.7896 \cdot 10^{-6}$ & $1.5336 \cdot 10^{-3}$ \\
1 & $10^{-9}$ & $2.9426 \cdot 10^{-7}$ & $6.4643 \cdot 10^{-7}$ & $1.5048 \cdot 10^{-6}$ \\
2 & $10^{-8}$ & $2.6024 \cdot 10^{-6}$ & $2.3678 \cdot 10^{-7}$ & $4.8565 \cdot 10^{-7}$ \\
3 & $10^{-7}$ & $3.9140 \cdot 10^{-6}$ & $1.8433 \cdot 10^{-6}$ & $1.1496 \cdot 10^{-4}$ \\
4 & $10^{-6}$ & $9.8001 \cdot 10^{-6}$ & $8.1611 \cdot 10^{-6}$ & $3.4538 \cdot 10^{-4}$ \\
5 & $10^{-5}$ & $3.885 \cdot 10^{-5}$ & $1.7317 \cdot 10^{-5}$ & $4.4141 \cdot 10^{-4}$ \\
6 & $10^{-4}$ & $1.5029 \cdot 10^{-4}$ & $9.3757 \cdot 10^{-5}$ & $3.7852 \cdot 10^{-4}$ \\
7 & $10^{-3}$ & $2.7077 \cdot 10^{-4}$ & $1.9412 \cdot 10^{-4}$ & $1.9051 \cdot 10^{-3}$ \\
8 & $10^{-2}$ & $4.0344 \cdot 10^{-4}$ & $2.5778 \cdot 10^{-4}$ & $1.2369 \cdot 10^{-3}$ \\
9 & $10^{-1}$ & $3.6802 \cdot 10^{-4}$ & $2.8245 \cdot 10^{-4}$ & $2.9078 \cdot 10^{-3}$ \\
10 & $10^{0}$ & $8.1445 \cdot 10^{-4}$ & $2.8836 \cdot 10^{-4}$ & $1.5894 \cdot 10^{-3}$ \\
11 & $10^{1}$ & $1.2358 \cdot 10^{-3}$ & $6.5678 \cdot 10^{-4}$ & $4.3943 \cdot 10^{-3}$ \\
12 & $10^{2}$ & $7.5575 \cdot 10^{-4}$ & $1.0798 \cdot 10^{-3}$ & $3.5281 \cdot 10^{-3}$ \\
13 & $10^{3}$ & $4.9746 \cdot 10^{-5}$ & $1.1125 \cdot 10^{-3}$ & $1.4208 \cdot 10^{-3}$ \\
14 & $10^{4}$ & $7.6975 \cdot 10^{-4}$ & $6.8704 \cdot 10^{-4}$ & $2.0413 \cdot 10^{-3}$ \\
15 & $10^{5}$ & $1.7023 \cdot 10^{-3}$ & $4.2663 \cdot 10^{-4}$ & $6.8348 \cdot 10^{-3}$ \\
16 & $10^{6}$ & $1.2327 \cdot 10^{-3}$ & $8.6765 \cdot 10^{-5}$ & $2.6735 \cdot 10^{-3}$ \\
17 & $10^{7}$ & $1.0743 \cdot 10^{-3}$ & $2.4247 \cdot 10^{-3}$ & $4.6575 \cdot 10^{-3}$ \\
18 & $10^{8}$ & $1.6157 \cdot 10^{-3}$ & $1.2343 \cdot 10^{-3}$ & $4.9646 \cdot 10^{-3}$ \\
19 & $10^{9}$ & $9.0416 \cdot 10^{-4}$ & $2.1804 \cdot 10^{-3}$ & $4.9958 \cdot 10^{-3}$ \\
\hline
\end{tabular}

Table 2: Free-volume model coefficients

\begin{tabular}{cccc}
\hline$B$ & $f_{0}$ & $\delta_{s}$ & $\kappa$ \\
\hline 1601.536 & 1.6913 & $4.5014 \cdot 10^{-1}$ & $5.5374 \cdot 10^{-1}$ \\
\hline
\end{tabular}

uniaxial and diaphragm tests considered in the previous step, since $\epsilon_{6}$ is zero in all of these tests. The optimized coefficients were obtained through the minimization of

$$
G(\kappa)=\sum_{j \in \subseteq}\left(\hat{y}_{j}(\kappa)-y_{j}\right)^{2}
$$

over uniaxial tests $\subseteq$ carried out at $T=[+10 ;-10 ;-30 ;-50]^{\circ} \mathrm{C}$ with the MD direction aligned at $45^{\circ}$ with respect to the loading axis ( $\mathrm{Li}$ et al. 2016). Using the optimal parameters obtained from the first step, namely $\left[B ; f_{0} ; \delta_{s} ; D_{13} ; D_{23}\right], D_{66}$ and $\kappa$ were calculated with fmincon minimization function.

The compliance coefficients $\left[D_{13} ; D_{23} ; D_{66}\right]$ obtained from the calibration procedure are presented in Table 1 while Table 2 shows the optimized free-volume model constants. The resulting master curves are reported in Fig. 6.

A comparison of the behavior of the current (superscript ' $c$ ') constitutive relations and the material model proposed by Li et al. (2016) (superscript 'p', ) has been conducted for all tests that have been employed in the aforementioned optimization procedure, namely $\mathcal{A}=\mathcal{U} \cup \mathfrak{D} \cup \mathfrak{S}$. The ratio between error norms of the two model predictions with respect to the experimental 


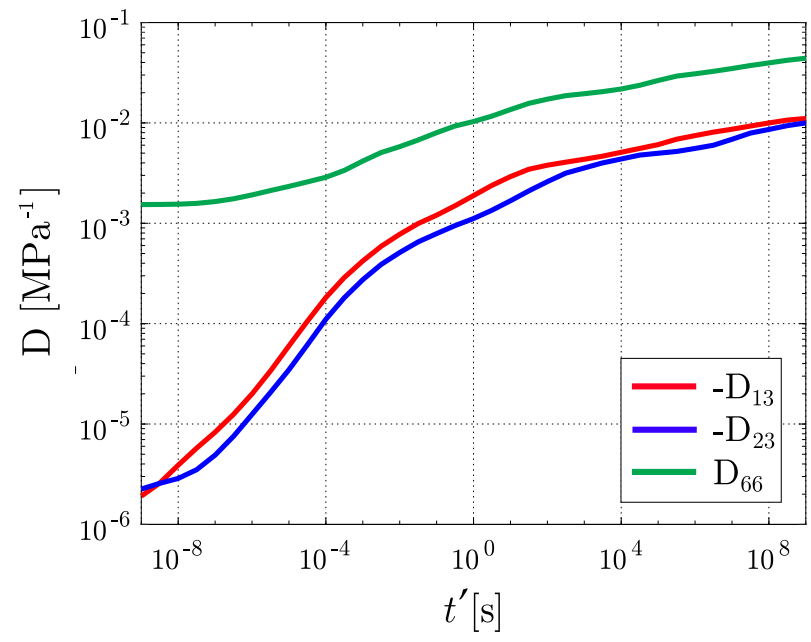

Figure 6: Creep compliance master curves $\left[-D_{13} ;-D_{23} ; D_{66}\right]$ reported as a function of the reduced time $t^{\prime}$, as obtained from the optimization procedure.

data points is

$$
\sum_{j \in \mathcal{A}} \frac{\left(\hat{y}_{j}^{r}\left(\boldsymbol{x}^{c}\right)-y_{j}\right)^{2}}{\left(\hat{y}_{j}^{p}\left(\boldsymbol{x}^{p}\right)-y_{j}\right)^{2}}=0.02
$$

indicating a $98 \%$ reduction of the error. The excellent improvement in the LLDPE material modeling is also shown in the following sections, where the nonlinear thermo-viscoelastic model is initially implemented into a finite element code and then it is adopted to numerically predict the behavior of the polymer over full field diaphragm inflation experiments and tensile, relaxation, and cyclic uniaxial tests.

\section{Finite element implementation}

The orthotropic generalized plane stress model presented in Sect. 2 has been implemented in the commercial finite element code Abaqus/Standard (v.6.14-1, Dassault Systèmes) through a user-defined material subroutine (UMAT). The numerical algorithm is suitable for any displacement-based finite element software and it can be easily adopted for the analysis of any thin film polymeric structure.

\subsection{Recursive Integration Algorithm}

The nonlinear thermo-viscoelastic constitutive relation represented by Eqs. (1)-(9) has to be discretized through a recursive algorithm, following Li et al. (2016). Taylor et al. (1970) proposed a method capable of minimizing the storage required to perform time integration for linear viscoelastic integrals. They showed that only history variables at the previous time step are necessary to determine the current stress state. Following this approach, several authors implemented the Schapery's nonlinear viscoelastic integral formulation (Henriksen, 1984; Lai and Bakker, 1996, Haj-Ali and Muliana, 2004).

Considering the Prony series representation of the compliance coefficients, Eq. (4), the thermo-viscoelastic constitutive relation in Eq. 9) can be rewritten for the multiaxial case as

$$
\epsilon_{i}^{t^{\prime}}=\alpha_{i}\left(T^{t^{\prime}}-T_{0}\right)+D_{i j, 0} \sigma^{t^{\prime}}+\sum_{k=1}^{n} D_{i j, k}\left(\sigma_{j}^{t^{\prime}}-\int_{0}^{t^{\prime}} e^{-\frac{t^{\prime}-s}{\tau_{k}}} \dot{\sigma}_{j}^{t^{\prime}} \mathrm{d} s\right),
$$

where the first term on the right-hand side is the thermal strain, while the remaining parts represent the mechanical deformation. The integral on the right-hand side constitutes the deformation history, and hence it is called hereditary integral $q_{j, k}^{t^{\prime}}$. It can be divided into two parts, from $t^{\prime}=0$ up to the previous time step $\left(0, t^{\prime}-\Delta t^{\prime}\right)$, and over the last time step $\left(t^{\prime}-\Delta t^{\prime}, t^{\prime}\right)$. Therefore, the recursive integration form becomes

$$
q_{j, k}^{t^{\prime}}=\int_{0}^{t^{\prime}-\Delta t^{\prime}} e^{-\frac{t^{\prime}-s}{\tau_{k}}} \dot{\sigma}_{j}^{t^{\prime}} \mathrm{d} s+\int_{t^{\prime}-\Delta t^{\prime}}^{t^{\prime}} e^{-\frac{t^{\prime}-s}{\tau_{k}}} \dot{\sigma}_{j}^{t^{\prime}} \mathrm{d} s .
$$

The first integral in Eq. 16 can be rewritten as a function of the hereditary integral $q_{j, k}^{t^{\prime}-\Delta t^{\prime}}$ for every term $k$ in the Prony series

$$
\int_{0}^{t^{\prime}-\Delta t^{\prime}} e^{-\frac{t^{\prime}-\Delta t^{\prime}-s}{\tau_{k}}} e^{-\frac{\Delta t^{\prime}}{\tau_{k}}} \dot{\sigma}_{j}^{t^{\prime}} \mathrm{d} s=e^{-\frac{\Delta t^{\prime}}{\tau_{k}}} q_{j, k}^{t^{\prime}-\Delta t^{\prime}}
$$

so that $q_{j, k}^{t^{\prime}-\Delta t^{\prime}}$ becomes a state variable to be updated and stored at the end of each time increment. Under the assumption that the shift parameter does not depend directly on time and the term $\sigma_{j}^{t^{\prime}}$ is linear over the current reduced time step increment $\Delta t^{\prime}$, the second integral in Eq. (16) can be expressed through integration by parts as

$$
\int_{t^{\prime}-\Delta t^{\prime}}^{t^{\prime}} e^{-\frac{t^{\prime}-s}{\tau_{k}}} \dot{\sigma}_{j}^{t^{\prime}} \mathrm{d} s=g\left(\Delta t^{\prime}, \tau_{k}\right)\left(\sigma_{j}^{t^{\prime}}-\sigma_{j}^{t^{\prime}-\Delta t^{\prime}}\right)
$$

where $g\left(\Delta t^{\prime}, \tau_{k}\right)=\tau_{k}\left(1-e^{-\frac{\Delta t^{\prime}}{\tau_{k}}}\right) / \Delta t^{\prime}$. Substituting Eqs. (17) and (18) into Eq. (16), the expression of the hereditary integral at the end of current reduced time $t^{\prime}$ has the expression

$$
q_{j, k}^{t^{\prime}}=e^{-\frac{\Delta t^{\prime}}{\tau_{k}}} q_{j, k}^{t^{\prime}-\Delta t^{\prime}}+g\left(\Delta t^{\prime}, \tau_{k}\right)\left(\sigma_{j}^{t^{\prime}}-\sigma_{j}^{t^{\prime}-\Delta t^{\prime}}\right) .
$$

Therefore, inserting Eq. (19) in Eq. (15), the total strain can be written as

$$
\epsilon_{i}^{t^{\prime}}=\tilde{D}_{i j, k}^{t^{\prime}} \sigma_{j}^{t^{\prime}}-f_{i, k}^{t^{\prime}-\Delta t^{\prime}}+\alpha_{i}\left(T^{t^{\prime}}-T_{0}\right)
$$

where

$$
\begin{aligned}
& \tilde{D}_{i j, k}^{t^{\prime}}=D_{i j, 0}+\sum_{k=1}^{n} D_{i j, k}-\sum_{k=1}^{n} D_{i j, k} g\left(\Delta t^{\prime}, \tau_{k}\right) \\
& f_{i, k}^{t^{\prime}-\Delta t^{\prime}}=\sum_{k=1}^{n} D_{i j, k}\left(e^{-\frac{\Delta t^{\prime}}{\tau_{k}}} q_{j, k}^{t^{\prime}-\Delta t^{\prime}}-g\left(\Delta t^{\prime}, \tau_{k}\right) \sigma_{j}^{t^{\prime}-\Delta t^{\prime}}\right)
\end{aligned}
$$

The reduced time increment $\Delta t^{\prime}$ is defined through Eq. (5) as

$$
\Delta t^{\prime}=\frac{\Delta t}{a_{t^{\prime}-\Delta t^{\prime}}}
$$

where the time shift factor $a^{t^{\prime}-\Delta t^{\prime}}$ is assumed constant over $\Delta t^{\prime}$ and from Eq. (6) is written as

$$
\log a^{t^{\prime}-\Delta t^{\prime}}=-\frac{B}{2.303 f_{0}}\left(\frac{\alpha_{v}\left(T-T_{0}\right)+\delta_{v} \theta^{t^{\prime}-\Delta t^{\prime}}+\delta_{s} \epsilon_{\mathrm{eff}}^{t^{\prime}-\Delta t^{\prime}}}{f_{0}+\alpha_{v}\left(T-T_{0}\right)+\delta_{v} \theta^{t^{\prime}-\Delta t^{\prime}}+\delta_{s} \epsilon_{\mathrm{eff}}^{t^{\prime}-\Delta t^{\prime}}}\right) .
$$



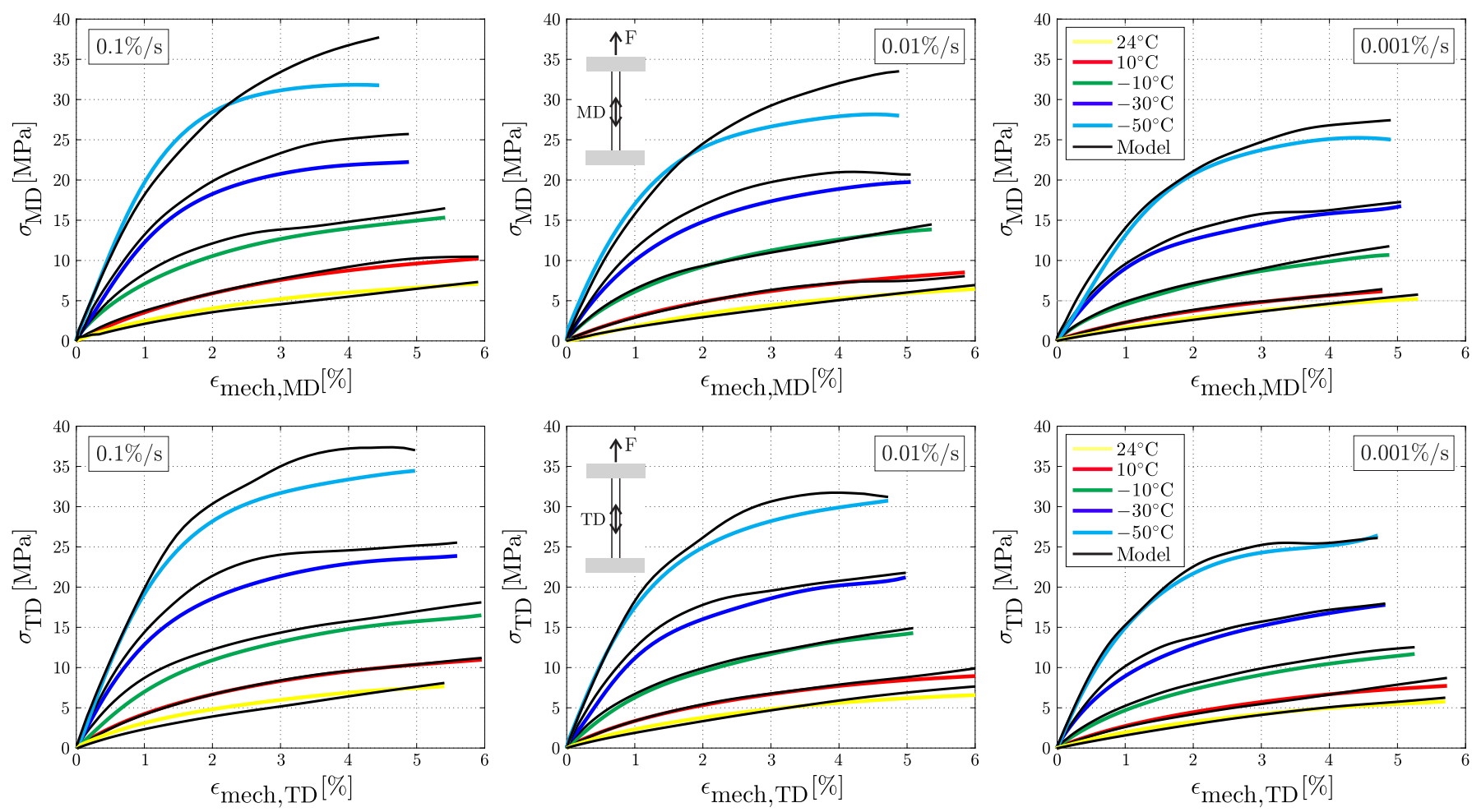

Figure 7: Comparison between experimental results and numerical predictions for uniaxial tension tests performed at $T=[+24 ;+10 ;-10 ;-30 ;-50]^{\circ} \mathrm{C}$ and $\dot{\epsilon}=[1,0.1,0.01 ; 0.001] \% \mathrm{~s}^{-1}$. Nominal stress vs. mechanical strain is reported for specimens aligned along MD (upper) and TD (lower).

Lastly, the mechanical dilatation $\theta^{t^{\prime}-\Delta t^{\prime}}$ and distorsion $\epsilon_{\mathrm{eff}}^{t^{\prime}-\Delta t^{\prime}}$, evaluated at the previous time step are

$$
\begin{aligned}
& \theta^{t^{\prime}-\Delta t^{\prime}}=\sum_{k=1}^{3} \epsilon_{k}^{t^{\prime}-\Delta t^{\prime}}, \\
& \epsilon_{\mathrm{eff}}^{t^{\prime}-\Delta t^{\prime}}=\sqrt{\frac{2}{3}\left[\sum_{k=1}^{3}\left(\epsilon_{k}^{t^{\prime}-\Delta t^{\prime}}-\frac{\theta^{t^{\prime}-\Delta t^{\prime}}}{3}\right)^{2}+\kappa\left(\epsilon_{6}^{t^{\prime}-\Delta t^{\prime}}\right)^{2}\right]} .
\end{aligned}
$$

\subsection{Architecture of user-defined material subroutine}

The role of the UMAT subroutine is to update the stress tensor $\sigma$ and the tangent stiffness matrix $\boldsymbol{C}$ at each iteration, after the Abaqus solver has passed to the UMAT several quantities available at the current time increment $\Delta t^{\prime}$, such as the strain increments $\Delta \boldsymbol{\epsilon}^{t^{\prime}}$ and the temperature $T_{t^{\prime}}$. Moreover, from the end of the previous time step $t^{\prime}-\Delta t^{\prime}, 139$ state variables (statev in Abaqus) have been stored and are available for the current increment. Those variables include the nominal stress $\sigma_{j}^{t^{\prime}-\Delta t^{\prime}}$, the hereditary integrals $q_{j, k}^{t^{\prime}-\Delta t^{\prime}}$, the mechanical dilatation $\theta^{t^{\prime}-\Delta t^{\prime}}$ and the distorsion $\epsilon_{\mathrm{eff}}^{t^{\prime}-\Delta t^{\prime}}$. Lastly, 150 user-specified material constants (props in Abaqus), presented in Tables 1, 2, A.1 and A.2, are passed to the subroutine.

The UMAT first calculates the reduced time shift factor $a^{t^{\prime}-\Delta t^{\prime}}$ from Eq. (23) and then the reduced time increment $\Delta t^{\prime}$ by means of Eq. 221. At a later time, once the engineering strains $\boldsymbol{\epsilon}^{\prime}$ are obtained, the current in-plane engineering stress tensor $\sigma^{t^{\prime}}$ is computed by inverting the constitutive equation 20 . Since the stress calculation is based on the time shift factor $a^{t^{\prime}-\Delta t^{\prime}}$, the time increment was always kept under a specified value in order to allow Abaqus to find an approximate equilibrium configuration at the end of each time increment. The accuracy of the solution has been verified by checking that the change in volumetric dilatation and distortion in all simulations remains below a specified tolerance. The out-of-plane strain $\epsilon_{3}^{t^{\prime}}$ is determined from $\sigma^{t^{\prime}}$, and therefore the volumetric dilatation $\theta^{t^{\prime}}$ and distortion $\epsilon_{\mathrm{eff}}^{t^{\prime}}$ at the current time step can be computed from Eq. 24) and stored together with the other history state variables (i.e. hereditary integrals). The computed stress tensor $\boldsymbol{\sigma}^{t^{\prime}}$ is returned to the Abaqus solver together with the tangent stiffness matrix.

\section{Validation and discussion}

An extensive and quantitative validation has been carried out in order to assess the robustness and accuracy of the developed nonlinear thermo-viscoelastic constitutive model for LLDPE. The validation is based on a comparison between experimental data and numerical predictions over different mechanical and thermal conditions, namely uniaxial and biaxial tests performed between room temperature $\left(24^{\circ} \mathrm{C}\right)$ and $-50^{\circ} \mathrm{C}$ and strain rates ranging from $1 \% \mathrm{~s}^{-1}$ to $0.001 \% \mathrm{~s}^{-1}$. The finite element results of the tests adopted in the calibration of material parameters serve as a validation of the numerical implementation and the pertinence of the fitted parameters. The comparison performed in the remaining experiments proves the reliability and precision of the constitutive relation. 


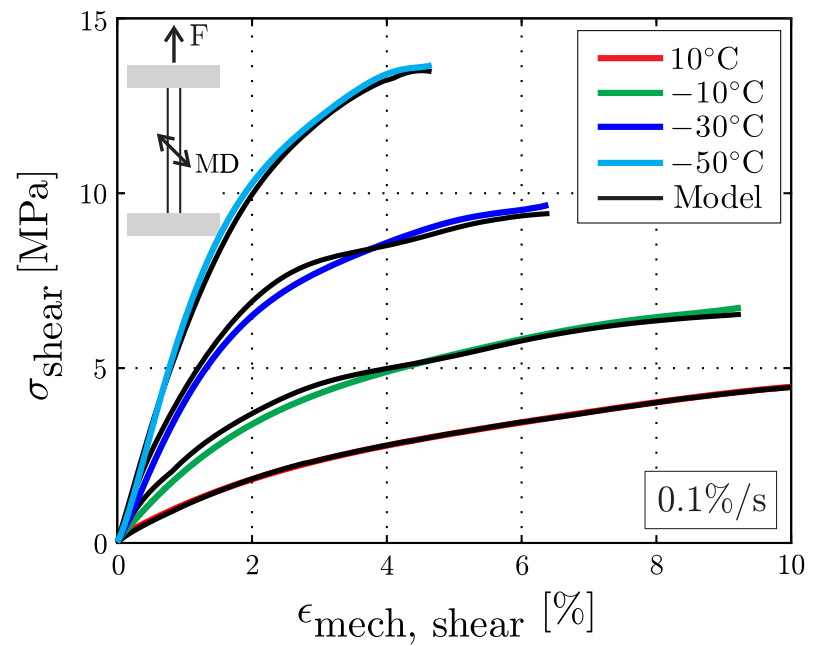

Figure 8: Shear stress vs. shear mechanical strain for uniaxial tension tests performed at $\dot{\epsilon}=0.1 \% \mathrm{~s}^{-1}$ and $T=[+10 ;-10 ;-30 ;-50]^{\circ} \mathrm{C}$. Experimental results from $\mathrm{Li}$ et al. (2016) are reported together with finite element predictions.

\subsection{Uniaxial tests}

The material parameters of the model have been calibrated employing only uniaxial tension tests performed at the lowest strain rate $\dot{\epsilon}=0.001 \% \mathrm{~s}^{-1}$. The following validation of the constitutive relation has been accomplished considering tensile, relaxation and cyclic tests carried out at faster deformation rates. The experimental setup and procedures of these experiments have been reported in Sect. 3.1. Numerical simulations were performed in Abaqus/Standard on a rectangular model of size 25.4 $\mathrm{mm} \times 200 \mathrm{~mm}$. The mesh consisted of M3D4 rectangular membrane elements, with the material properties described in Sect. 4 The interface between Abaqus and the UMAT subroutine was established through the command *user material, specifying the number of state variables with *depvar. A material coordinate system was defined on all elements through *orientation. The bottom nodes were pinned, while the upper nodes were constrained in the horizontal direction, with an imposed vertical displacement that matches the nominal strain rate of the test.

\subsubsection{Tensile tests}

Results from the numerical predictions of the tensile tests performed at $T=[+24 ;+10 ;-10 ;-30 ;-50]^{\circ} \mathrm{C}$ and $\dot{\epsilon}=$ $[1,0.1,0.01 ; 0.001] \% \mathrm{~s}^{-1}$ are compared with experimental measurements in Fig. 7. Although only the data at the lowest deformation rate had been employed in the determination of the material parameters, an excellent agreement can be noticed throughout the whole range of strain rates investigated. Therefore, the comparison performed at $\dot{\epsilon}=0.001 \% \mathrm{~s}^{-1}$ proves the precision of the fitted parameters and the numerical implementation, while the tests at higher strain rates show the accuracy of the constitutive relation. From Fig. 7] it can be clearly seen that the material becomes stiffer with an increase of strain rate or a decrease of the temperature.

The behavior of the proposed model in shear has been compared to uniaxial tensile experiments performed by $\mathrm{Li}$ et al.

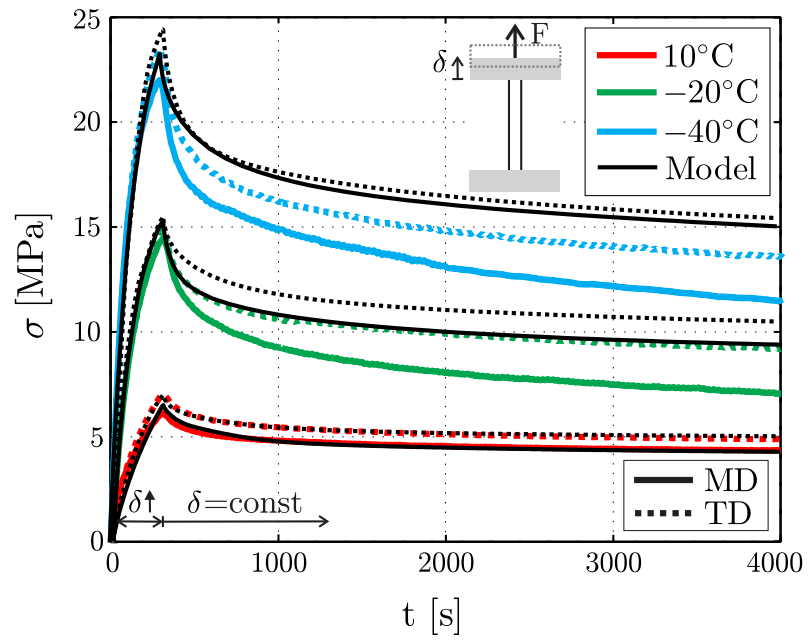

Figure 9: Comparison between experimental results and numerical predictions for relaxation tests carried out at $T=[+10 ;-20 ;-40]^{\circ} \mathrm{C}$ for both MD (solid line) and TD (dashed line). The evolution of the stress with time shows the first phase of tensile loading for $\mathrm{t} \leq 300 \mathrm{~s}$ and the second phase of stress relaxation at constant strain until $\mathrm{t}=4000 \mathrm{~s}$.

(2016) in order to judge the correctness of the fitted parameters $D_{66}$ and $\kappa$. These tests were carried out on a sample having the machine direction (MD) oriented at $45^{\circ}$ with respect to the loading direction. Figure 8 shows a very good agreement between the shear stresses and strains at $\dot{\epsilon}=0.1 \% \mathrm{~s}^{-1}$ and different temperatures, namely $T=[+10 ;-10 ;-30 ;-50]^{\circ} \mathrm{C}$.

The constitutive relation has been proven to be accurate also for $25.4 \mu \mathrm{m}$ and $50.8 \mu \mathrm{m}$ thick samples. Uniaxial tensile tests were performed as the same temperatures and strain rates, but are not reported here for conciseness.

\subsubsection{Relaxation tests}

A comparison between experiments and simulations for relaxation tests at $T=[+10 ;-20 ;-40]^{\circ} \mathrm{C}$ is reported in Fig. 9 for both MD and TD. This set of tests allows the evaluation of the constitutive relation performance under a long-term relaxation regime, a condition which has not been used in the determination of the model parameters. However, it should be noted that even though creep and relaxation are caused by the same molecular mechanism, the relaxation response moves faster toward its equilibrium value and therefore some differences between the model predictions and experiments can be expected because in the present study the in-plane creep compliance master curves have been obtained only through creep tests.

The first phase of the tests, where the film is subjected to a deformation rate of $0.01 \% \mathrm{~s}^{-1}$ up to $3 \%$ of mechanical strain, shows an excellent agreement. In the second phase, the model provides a good prediction of the experimental measurements under condition of stress relaxation. At low temperatures, the model is stiffer compared to the experimental measurements, with a maximum error of $28 \%$ at $-40^{\circ} \mathrm{C}$. 


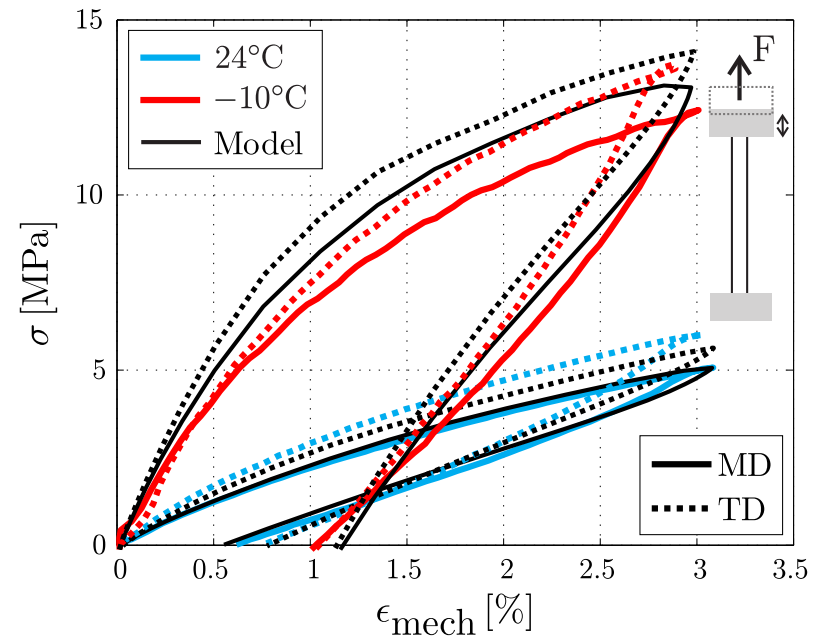

Figure 10: Nominal stress vs. mechanical strain for uniaxial loading and unloading tension tests at $\dot{\epsilon}=0.1 \% \mathrm{~s}^{-1}$. Experimental results and simulations performed for both MD (solid) and TD (dashed) at $24^{\circ} \mathrm{C}$ (blue) and $-10^{\circ} \mathrm{C}$ (red).
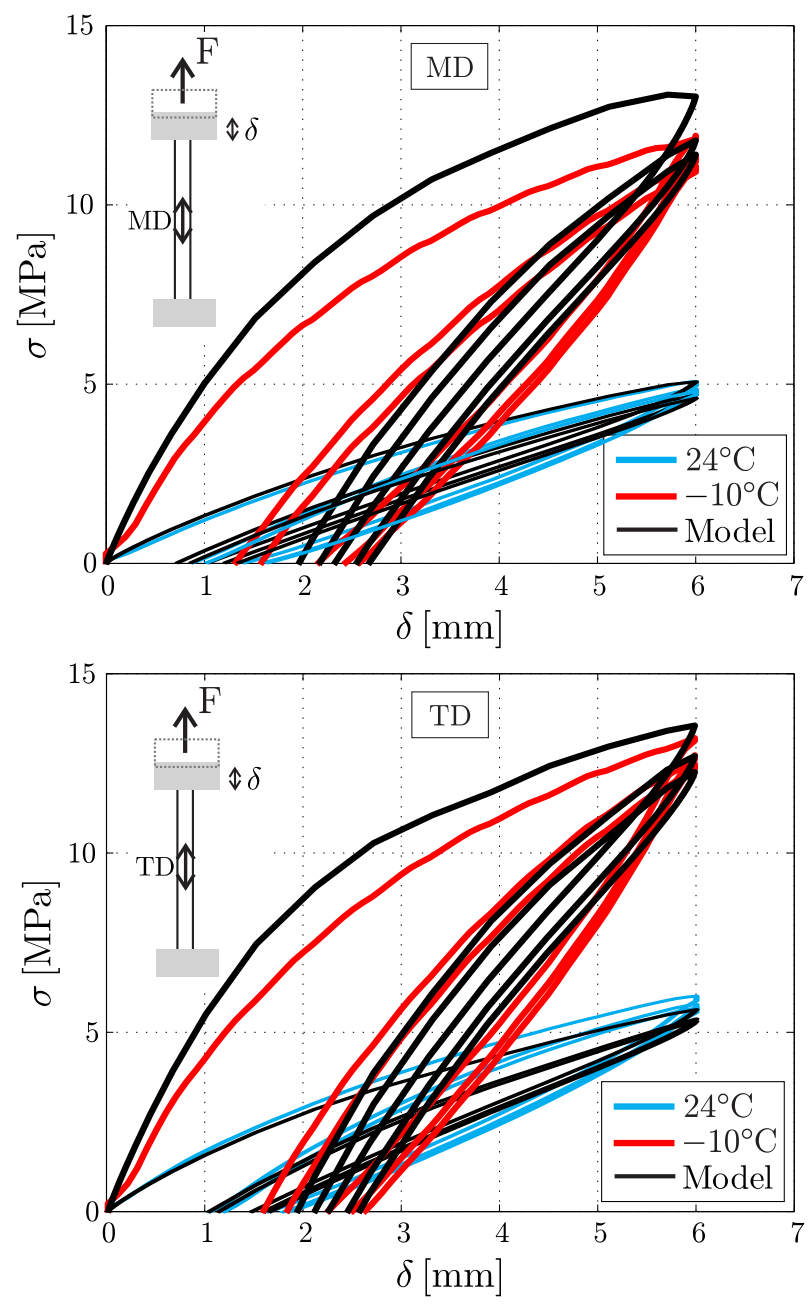

Figure 11: Uniaxial cyclic tests performed at a strain rate of $0.1 \% \mathrm{~s}^{-1}$ up to $3 \%$ of mechanical strain, at $24^{\circ} \mathrm{C}$ (blue) and $-10^{\circ} \mathrm{C}$ (red). Longitudinal nominal stress vs. longitudinal displacement is reported for MD (upper) and TD (lower).

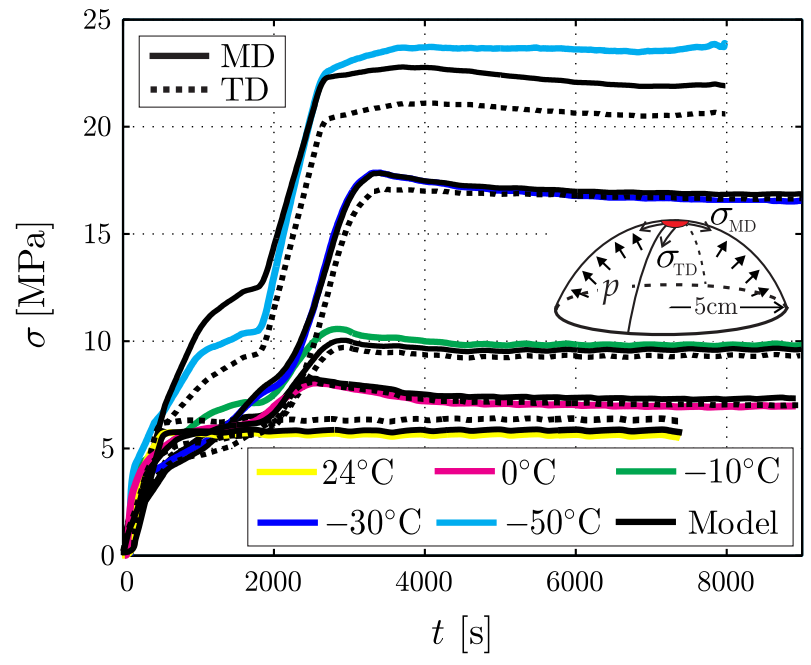

Figure 12: Evolution of stress as a function of time for long-duration diaphragm inflation tests of a circular membrane at different temperatures, namely $T=[+24 ; 0 ;-10 ;-30 ;-50]^{\circ} \mathrm{C}$. The experimental nominal stress at the apex of the diaphragm is calculated through Eq. 10, whereas finite element analysis predicts stresses along MD (solid curve) and TD (dashed curve).

\subsubsection{Cyclic tests}

The ability of the constitutive equation of modeling unloading behavior, stress softening, and hysteresis has been assessed by means of cyclic tension tests run at a mechanical strain rate of $0.1 \% \mathrm{~s}^{-1}$ at room temperature $\left(24^{\circ} \mathrm{C}\right)$ and at $-10^{\circ} \mathrm{C}$. In Fig. 10 , the finite element predictions of nominal stress vs. mechanical strain have been superimposed to experimental measurements for the first cycle at both temperatures and sample loading directions. It can be observed that hysteresis increases at lower temperatures. For the same experiments and numerical analyses, Fig. 11 shows stress evolution as a function of the imposed displacement over three cycles. It can be concluded that the proposed constitutive relation correctly predicts the material behavior under multiple cycles. In fact, it is able to capture both stress softening and hysteresis without the implementation of a switching rule between loading and unloading, as previously suggested (Xia et al. 2006).

\subsection{Diaphragm inflation tests}

The accuracy of the proposed model has been investigated through a comparison between numerical predictions and experimental results of long-term diaphragm inflation tests described in Sect. 3.2.

A finite element model of a $100 \mathrm{~mm}$ diameter membrane was set up in Abaqus/Standard. Because of symmetry, only one quarter of the test sample was modeled through a mesh of M3D3 triangular membrane elements, which were given the material properties described in Sect. 4. Pinned boundary condition were assigned to the circular edge of the film, which was partially sandwiched between two closing aluminum rings of thickness $10 \mathrm{~mm}$ and width $12.5 \mathrm{~mm}$. These upper and lower rings were modeled with C3D8 solid elements in order to take into account any thermal stresses that may arise during cooling because of the 

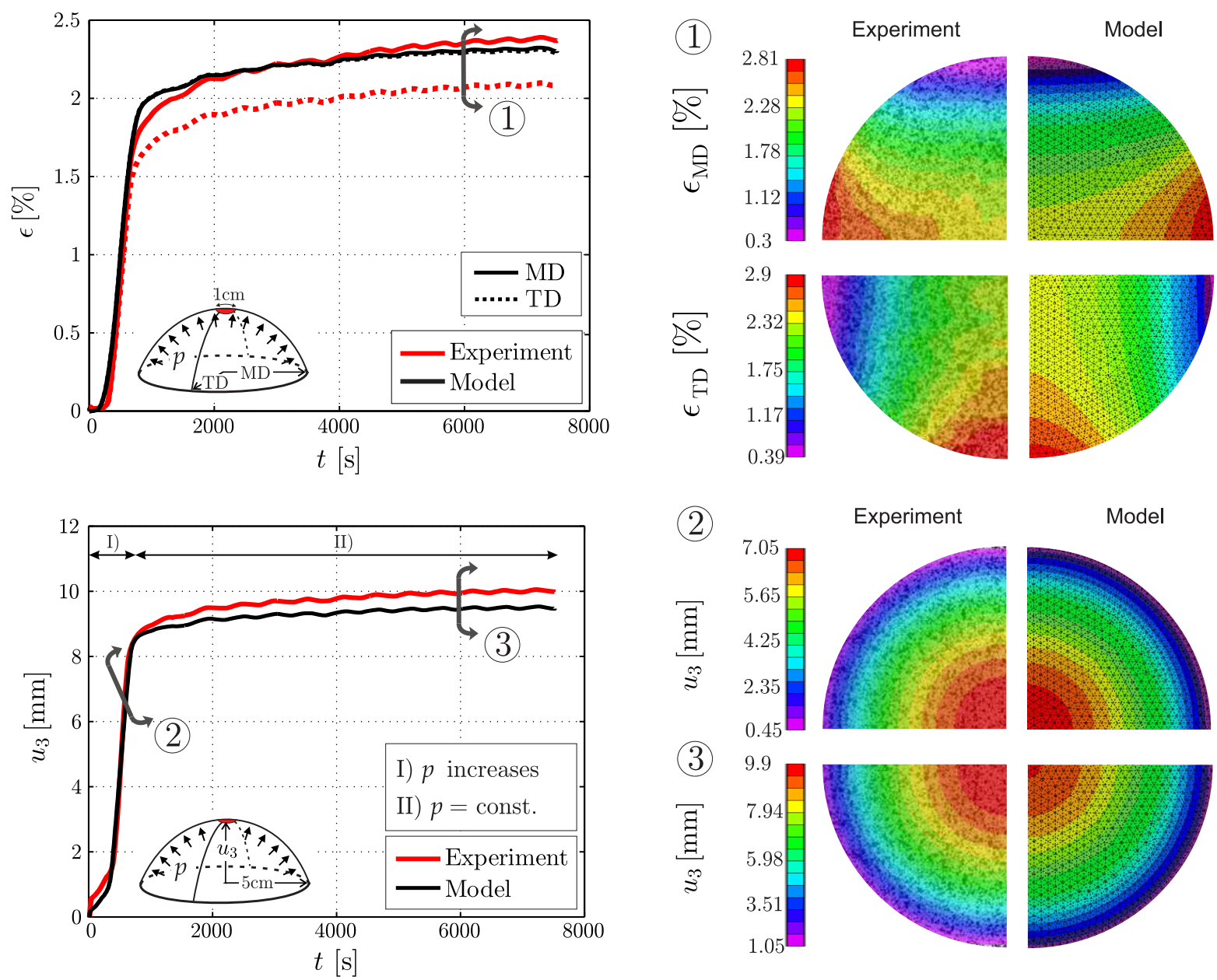

Figure 13: Comparison between experimental results and finite element simulations of diaphragm inflation tests at room temperature $\left(+24^{\circ} \mathrm{C}\right)$. Upper part: total engineering strain extracted from the apex of the diaphragm (strains were averaged on the red circular area of diameter $1 \mathrm{~cm}$ reported in the inset) is reported as a function of time (left), while a comparison of the total strain distributions at $\mathrm{t}=6000 \mathrm{~s}$ (1) is reported over the whole circular membrane for both MD and TD (right). Lower part: vertical displacement of membrane's apex vs. time (left) and contour plot of out-of-plane displacements throughout the whole diaphragm during loading (2) and at constant pressure (3).

mismatch between aluminum and polyethylene CTEs. Surfaceto-surface contact between the film and the rings was modeled through the *contact pair function in Abaqus, employing a hard surface normal behavior and a frictionless tangential contact (friction coefficients of 0.2 and 0.5 were considered, without any significant difference in the results). The interface between Abaqus and the UMAT subroutine was established through the command *user material, specifying the number of state variables with *depvar. A geometrically nonlinear analysis was carried out. The material coordinate system was defined on all membrane elements through the *orientation function. The experimental temperature and pressure profiles were defined using *temperature and *dsload commands.

An initial stage of the analysis was required to stabilize the membrane when a small initial pressure is applied perpendicular to the membrane (Wong and Pellegrino, 2006). This stabilization phase was achieved by imposing a uniform tiny user-defined initial stress on all elements, using *initial condition,type=solution, user and the related subroutines sdvini (to initialize state de- pendent variables) or sigini (to impose an initial stress field). After the application of the initial pre-stress, a static step was performed in order to achieve equilibrium. Other strategies, such as prescribing an infinitesimal set of edge radial displacements or imposing an initial small decrease of temperature were found to lead to the same results.

Since the stress at the apex of the inflated membrane has been assumed isotropic in the calibration of the material parameters reported in Tables 1 and 2, a validation of this assumption is presented in Fig. 12. Indeed, it can be observed that the maximum difference between the numerical prediction of the stresses along MD and TD is ca. $10 \%$, and they match very well the experimental data obtained through Eq. (10). The excellent comparison between numerical predictions and experimental measurements in Fig. 12 validates the optimization procedure of the material parameter and the finite element implementation of the constitutive relation.

Furthermore, a detailed comparison between numerical results and experimental measurements is reported in Figs. 13 and 14 

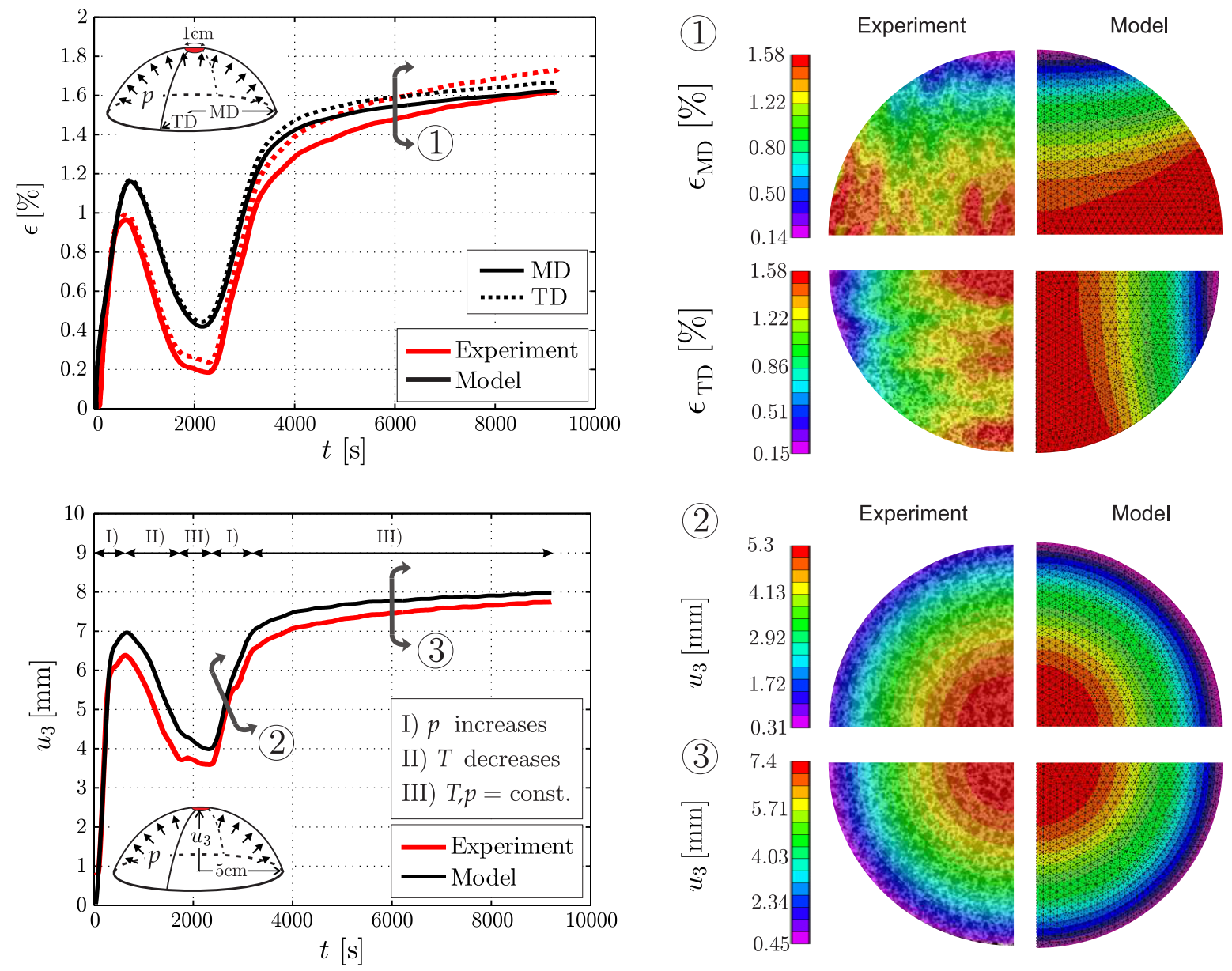

Figure 14: Comparison between experimental results and finite element simulations of diaphragm inflation tests at $-30^{\circ} \mathrm{C}$. Upper part: total engineering strain extracted from the apex of the diaphragm is reported as a function of time (left), while a comparison of the total strain distribution at $t=6000 \mathrm{~s}$ (1) is reported over the whole circular membrane for both MD and TD (right). Lower part: vertical displacement of membrane's apex vs. time (left) and contour plot of out-of-plane displacements throughout the whole diaphragm during loading (2) and at constant pressure (3).

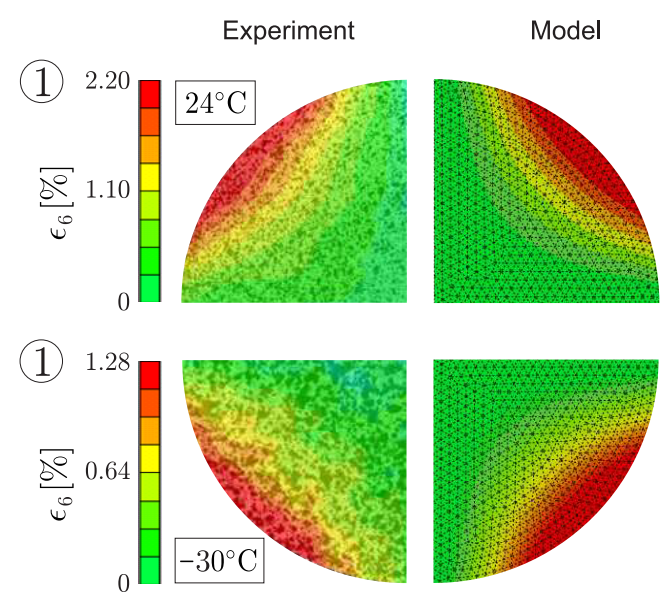

Figure 15: Shear strain $\epsilon_{6}$ distribution in a quarter of the circular membrane during long-duration inflation experiments. Comparison between experimental measurements (left) and finite element results (right) at $t=6000 \mathrm{~s}$ are reported for temperatures of $+24^{\circ} \mathrm{C}$ and $-30^{\circ} \mathrm{C}$. Snapshots are taken at the instant numbered with 1 in Fig. 13 and Fig. 14
The variation with time of the in-plane total strains and outof-plane displacements are presented for a point at the apex of the diaphragm. An excellent agreement can be noticed, with a maximum error of $15 \%$. In the same figures, a comparison of strains and displacement fields throughout the whole membrane is shown on the right. These contour plots confirm that the model is able to correctly predict the strain and displacement distributions in the whole structure.

The accuracy of the nonlinear thermo-viscoelastic model has been further proved with the comparison reported in Fig. 15. The plot shows the experimentally measured shear strain distribution throughout the membrane and its numerical prediction, at $t=$ $6000 \mathrm{~s}$ for tests performed at $+24^{\circ} \mathrm{C}$ and $-30^{\circ} \mathrm{C}$. A very good agreement between experiments and simulations is achieved in all diaphragm tests performed, even if for conciseness only the results at $+24^{\circ} \mathrm{C}$ and $-30^{\circ} \mathrm{C}$ have been presented here.

Therefore, through the model validation the effectiveness of the numerical implementation, the accuracy of the fitted parameters and the proposed constitutive relation have been demonstrated. LLDPE film behavior has been predicted with a maximum error of ca. $28 \%$ over a variety of different tests, strain rates 
spanning from $0.001 \% \mathrm{~s}^{-1}$ to $1 \% \mathrm{~s}^{-1}$, and temperatures between $+24^{\circ} \mathrm{C}$ and $-50^{\circ} \mathrm{C}$.

The maximum discrepancy between the model predictions and experiments is observed at lower temperatures during relaxation tests since such loading condition was considered neither in the in-plane compliance master curves determination nor in the parameters calibration. Overall it can be noted that the model slightly loses accuracy when the temperature decreases and approaches the glass transition temperature, where a change in the molecular response mechanism due to morphological factors occurs and the time shift factor through the free volume model becomes less precise.

\section{Conclusions}

A nonlinear thermo-viscoelastic constitutive relation for thin polymeric film based on hereditary integral representation for multiaxial deformation has been presented, implemented in a finite element code, and experimentally validated against different tests covering a wide range of temperatures, strain rates and mechanical loading conditions. The proposed model relies on the definition of the out-of-plane compliances, necessary to describe the mechanical dilatation of the material expressed in the free volume theory of nonlinear viscoelasticity. Because of the difficulty of following the current strain during experiments, the present formulation relates the engineering strain and stress tensors.

Low deformation rate uniaxial tension tests and biaxial inflation experiments have been performed at several temperatures in order to calibrate the constitutive parameters. The resulting model has been discretized through a recursive algorithm and implemented into the finite element code Abaqus/Standard by means of a user-defined subroutine.

Furthermore, the constitutive relation has been experimentally validated against data obtained from diaphragm inflation tests and uniaxial tension, relaxation and cyclic tests, showing an excellent agreement for temperatures between $24^{\circ} \mathrm{C}$ and $-50^{\circ} \mathrm{C}$, and for strain rates that spans four orders of magnitude, from $0.001 \% \mathrm{~s}^{-1}$ to $1 \% \mathrm{~s}^{-1}$. This high fidelity nonlinear thermoviscoelastic model will be employed in the structural design of super-pressure balloons in order to correctly understand the film behavior during flight, especially where high stress concentration appear, such as near the tendons or near the apex of the balloon.

The presented mathematical formulation, experimental campaign, numerical implementation and validation, were focused on linear-low density polyethylene (LLDPE) film.

However, the same approach is applicable to the development of plane stress constitutive models for any thermo-rheologically simple materials that show time-dependent effects, where high prediction accuracy is needed.

To conclude, the present modeling might be enhanced in different directions, for example visco-plastic effects could be considered after a yield locus, considerably dependent on temperature and strain rate, will be calibrated.
David Wakefield (Tensys Limited) and Dr. James Rand (Winzen Engineering) for helpful comments and discussions. Financial support from the NASA Balloon Program Office is gratefully acknowledged.

\section{AppendixA. Material parameters}

The following material parameters were taken from previous studies. The polynomial coefficients for the in-plane CTEs obtained by Young (2010) are presented in Table A.1.

Table A.1: Polynomial coefficients of in-plane CTEs

\begin{tabular}{crr}
\hline $\mathbf{j}$ & $p_{1, k}\left[\mathrm{~K}^{-1}\right]$ & $p_{2, k}\left[\mathrm{~K}^{-1}\right]$ \\
\hline 0 & $-2.069691 \cdot 10^{1}$ & $1.720772 \cdot 10^{1}$ \\
1 & $1.183351 \cdot 10^{0}$ & $-1.010550 \cdot 10^{0}$ \\
2 & $-3.065068 \cdot 10^{-2}$ & $2.692250 \cdot 10^{-2}$ \\
3 & $4.755522 \cdot 10^{-4}$ & $-4.302432 \cdot 10^{-4}$ \\
4 & $-4.922242 \cdot 10^{-6}$ & $4.593443 \cdot 10^{-6}$ \\
5 & $3.580707 \cdot 10^{-8}$ & $-3.451674 \cdot 10^{-8}$ \\
6 & $-1.877228 \cdot 10^{-10}$ & $1.871957 \cdot 10^{-10}$ \\
7 & $7.146794 \cdot 10^{-13}$ & $-7.383200 \cdot 10^{-13}$ \\
8 & $-1.961127 \cdot 10^{-15}$ & $2.102008 \cdot 10^{-15}$ \\
9 & $3.783162 \cdot 10^{-18}$ & $-4.213295 \cdot 10^{-18}$ \\
10 & $-4.870561 \cdot 10^{-21}$ & $5.644505 \cdot 10^{-21}$ \\
11 & $3.757961 \cdot 10^{-24}$ & $-4.538553 \cdot 10^{-24}$ \\
12 & $-1.314349 \cdot 10^{-27}$ & $1.656629 \cdot 10^{-27}$ \\
\hline
\end{tabular}

The in-plane compliance master curves are reported in Fig. A.16, while their Prony series coefficients $\left[D_{11} ; D_{12} ; D_{22}\right]$, determined through creep tests by Li et al. (2016), are listed in Table A.2.

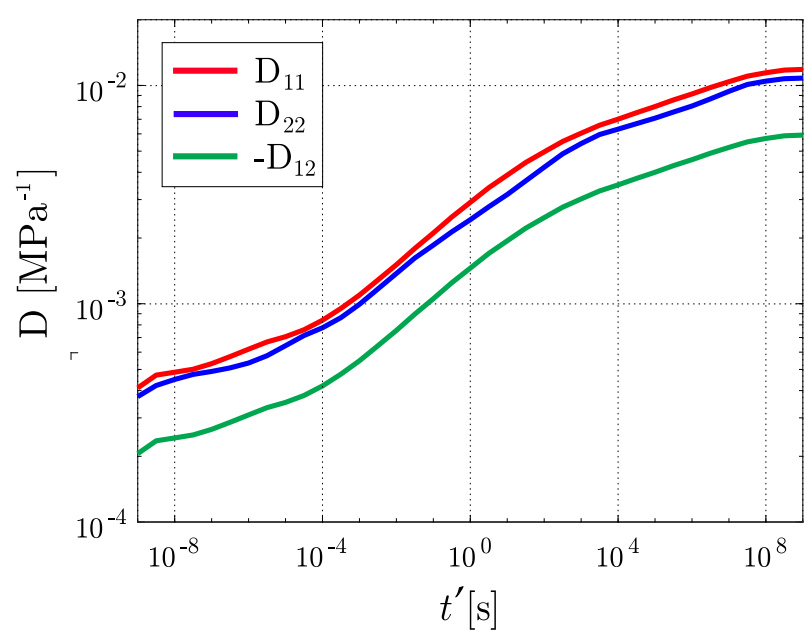

Figure A.16: In-plane creep compliance master curves $\left[D_{11} ;-D_{12} ; D_{22}\right]$ reported as a function of the reduced time $t^{\prime}$. 
Table A.2: In-plane Prony coefficients for compliance matrix $\boldsymbol{D}\left[\mathrm{MPa}^{-1}\right]$

\begin{tabular}{ccccc}
\hline $\mathbf{j}$ & $\tau[\mathrm{s}]$ & $D_{11, j}$ & $-D_{12, j}$ & $D_{22, j}$ \\
\hline 0 & 0 & $3.0 \cdot 10^{-4}$ & $1.5 \cdot 10^{-4}$ & $3.0 \cdot 10^{-4}$ \\
1 & $10^{-9}$ & $1.7427 \cdot 10^{-4}$ & $8.7137 \cdot 10^{-5}$ & $1.0998 \cdot 10^{-4}$ \\
2 & $10^{-8}$ & $6.5109 \cdot 10^{-6}$ & $3.2555 \cdot 10^{-6}$ & $5.8649 \cdot 10^{-5}$ \\
3 & $10^{-7}$ & $6.2843 \cdot 10^{-5}$ & $3.1421 \cdot 10^{-5}$ & $2.4143 \cdot 10^{-5}$ \\
4 & $10^{-6}$ & $1.0754 \cdot 10^{-4}$ & $5.3772 \cdot 10^{-5}$ & $4.2218 \cdot 10^{-5}$ \\
5 & $10^{-5}$ & $5.8577 \cdot 10^{-5}$ & $2.9289 \cdot 10^{-5}$ & $1.5063 \cdot 10^{-4}$ \\
6 & $10^{-4}$ & $1.5508 \cdot 10^{-4}$ & $7.7540 \cdot 10^{-5}$ & $9.6093 \cdot 10^{-5}$ \\
7 & $10^{-3}$ & $2.8439 \cdot 10^{-4}$ & $1.4219 \cdot 10^{-4}$ & $2.5980 \cdot 10^{-4}$ \\
8 & $10^{-2}$ & $4.5672 \cdot 10^{-4}$ & $2.2836 \cdot 10^{-4}$ & $4.4622 \cdot 10^{-4}$ \\
9 & $10^{-1}$ & $6.4614 \cdot 10^{-4}$ & $3.2307 \cdot 10^{-4}$ & $4.7934 \cdot 10^{-4}$ \\
10 & $10^{0}$ & $8.6980 \cdot 10^{-4}$ & $4.3490 \cdot 10^{-4}$ & $5.9115 \cdot 10^{-4}$ \\
11 & $10^{1}$ & $1.0174 \cdot 10^{-3}$ & $5.0872 \cdot 10^{-4}$ & $7.5112 \cdot 10^{-4}$ \\
12 & $10^{2}$ & $1.1201 \cdot 10^{-3}$ & $5.6007 \cdot 10^{-4}$ & $1.2374 \cdot 10^{-3}$ \\
13 & $10^{3}$ & $1.0881 \cdot 10^{-3}$ & $5.4407 \cdot 10^{-4}$ & $1.2622 \cdot 10^{-3}$ \\
14 & $10^{4}$ & $8.6245 \cdot 10^{-4}$ & $4.3123 \cdot 10^{-4}$ & $6.4408 \cdot 10^{-4}$ \\
15 & $10^{5}$ & $1.0593 \cdot 10^{-3}$ & $5.2967 \cdot 10^{-4}$ & $8.2460 \cdot 10^{-4}$ \\
16 & $10^{6}$ & $1.1495 \cdot 10^{-3}$ & $5.7473 \cdot 10^{-4}$ & $9.4359 \cdot 10^{-4}$ \\
17 & $10^{7}$ & $1.4144 \cdot 10^{-3}$ & $7.0721 \cdot 10^{-4}$ & $1.7422 \cdot 10^{-3}$ \\
18 & $10^{8}$ & $9.2623 \cdot 10^{-4}$ & $4.6312 \cdot 10^{-4}$ & $7.7978 \cdot 10^{-4}$ \\
19 & $10^{9}$ & $1.3703 \cdot 10^{-4}$ & $6.8515 \cdot 10^{-5}$ & $9.7438 \cdot 10^{-5}$ \\
\hline
\end{tabular}

\section{References}

Bergstrom, J., Boyce, M., 2001. Constitutive modeling of the time-dependent and cyclic loading of elastomers and application to soft biological tissues. Mech. Mater. 33, 523-530.

Brinson, H. F., Brinson, L. C., 2008. Polymer engineering science and viscoelasticity. An introduction. Springer.

Cathey, H. J., 2009. The nasa super pressure balloon - a path to flight. Adv. Space Res. 44, 23-38.

Deng, X., Pellegrino, S., 2011. A technique to predict clefting of lobed superpressure balloons. pp. AIAA-2011-6830.

Dupaix, R., Boyce, M., 2007. Constitutive modeling of the finite strain behavior of amorphous polymers in and above the glass transition. Mech. Mater. 39, 39-52.

Haj-Ali, R., Muliana, A., 2004. Numerical finite element formulation of the schapery nonlinear viscoelastic material model. Int. J. Numer. Meth. Eng. 59, 25-45.

Henriksen, M., 1984. Nonlinear viscoelastic stress analysis - a finite element approach. Comput. Struct. 18, 133-139.

Knauss, W., Emri, I., 1981. Nonlinear viscoelasticity based on free volume consideration. Comput. Struct. 13, 123-128.

Knauss, W., Emri, I., 1987. Volume change and the nonlinearity thermoviscoelastic constitution of polymers. Polym. Eng. Sci. 27, 86-100.

Lai, J., Bakker, A., 1996. 3-d schapery representation for nonlinear viscoelasticity and finite element implementation. Comput. Mech. 18, 182-191.

Li, J., Kwok, K., Pellegrino, S., 2016. Thermoviscoelastic models for polyethylene thin films. Mech. Time-Depend. Mat. 13 (1), 13-43.

Pagitz, M., Pellegrino, S., 2010. Maximally stable lobed balloons. Int. J. Solids Structures 47, 1496-1507.

Park, S., Kim, Y., 2001. Fitting prony-series viscoelastic models with power-law presmoothing. J. Mater. Civil Eng. 13 (1), 26-32.

Rand, J., Henderson, J., Grant, D., 1996. Nonlinear behavior of linear lowdensity polyethylene. Polym. Eng. Sci. 36, 1058-1064.

Rand, J., Sterling, W., 2006. A constitutive equation for stratospheric ballon materials. Adv. Space Res. 37, 2087-2091.

Ree, T., Eyring, H., 1955. Theory for non-newtonian flow. i. solid plastic system. J. Appl. Phys. 26, 793.
Reu, P., 2012. Introduction to digital image correlation: best practices and applications. Exp. Techniques 36 (1), 3-4.

Schapery, R., 1966. An engineering theory of nonlinear viscoelasticity with applications. Int. J. Solids Structures 2, 407-425.

Schapery, R., 1969. On the characterization of nonlinear viscoelastic materials. Polym. Eng. Sci. 9, 295-310.

Smith, I., Rainwater, E., 2004. Optimum designs for superpressure balloons. Adv. Space Res. 33, 16881693

Sutton, M., Orteu, J., Schreier, H., 2009. Image correlation for shape, motion and deformation measurements: basic concepts, theory and applications. Springer.

Taylor, R., Pister, K., Goudreau, G., 1970. Thermomechanical analysis of viscoelastic solids. Int. J. Numer. Meth. Eng. 2, 45-59.

Wakefield, D., 2005. Numerical modeling of pumpkin balloon instability. pp. AIAA-2005-7445.

Wong, Y., Pellegrino, S., 2006. Wrinkled membranes. part iii: numerical simulations. J. Mech. Mater. Struct. 1 (1), 63-95.

Xia, Z., Shen, X., Ellyin, F., 2006. An assessment of nonlinearly viscoelastic constitutive models for cyclic loading: the effect of a general loading/unloading rule. Mech. Time-Depend. Mat. 9, 281-300.

Yoon, K., Park, C., 2000. Stability of a two-layer blown film coextrusion. J. Non-Newtonian Fluid Mech. 89, 97-116.

Young, L., 2010. Cte curve fitting. In: NASA Balloon Program Office report. 